\title{
Periodicities of T-systems and Y-systems, Dilogarithm Identities, and Cluster Algebras II: Types $C_{r}, F_{4}$, and $G_{2}$
}

\author{
by \\ Rei Inoue, Osamu Iyama, Bernhard Keller, Atsuo Kuniba and \\ Tomoki NAKANISHI
}

\begin{abstract}
We prove the periodicities of the restricted T-systems and $\mathrm{Y}$-systems associated with the quantum affine algebra of type $C_{r}, F_{4}$, and $G_{2}$ at any level. We also prove the dilogarithm identities for these Y-systems at any level. Our proof is based on the tropical Y-systems and the categorification of the cluster algebra associated with any skew-symmetric matrix by Plamondon.

2010 Mathematics Subject Classification: Primary 13F60; Secondary 17B37.

Keywords: T-systems, Y-systems, cluster algebra, dilogarithm.

\section{Contents}

1 Introduction 44

2 Main results 44

3 Type $C_{r} \quad 50$

4 Type $F_{4} \quad 66$

5 Type $G_{2} \quad 74$

6 Mutation equivalence of quivers 84

References 84

Communicated by H. Nakajima. Received April 8, 2010.

R. Inoue: Faculty of Pharmaceutical Sciences, Suzuka University of Medical Science, Suzuka, 513-8670, Japan;

e-mail: reiiy@math.s.chiba-u.ac.jp

O. Iyama: Graduate School of Mathematics, Nagoya University, Nagoya, 464-8604, Japan;

e-mail: iyama@math.nagoya-u.ac.jp

B. Keller: Université Paris Diderot - Paris 7, UFR de Mathématiques, Institut de Mathématiques de Jussieu, UMR 7586 du CNRS, Case 7012, 2, place Jussieu, 75251 Paris Cedex 05, France; e-mail: keller@math.jussieu.fr

A. Kuniba: Institute of Physics, University of Tokyo, Tokyo, 153-8902, Japan;

e-mail: atsuo@gokutan.c.u-tokyo.ac.jp

T. Nakanishi: Graduate School of Mathematics, Nagoya University, Nagoya, 464-8604, Japan; e-mail: nakanisi@math.nagoya-u.ac.jp
\end{abstract}




\section{$\S 1$. Introduction}

This is a continuation of the paper [IIKKN]. In [IIKKN], we proved the periodicities of the restricted T-systems and Y-systems associated with the quantum affine algebra of type $B_{r}$ at any level. We also proved the dilogarithm identities for these Y-systems at any level. Our proof was based on the tropical Y-systems and the categorification of the cluster algebra associated with any skew-symmetric matrix by Plamondon [P1, P2]. In this paper, using the same method, we prove the corresponding statements for types $C_{r}, F_{4}$, and $G_{2}$, thereby completing all the non-simply laced types.

The results are basically parallel to type $B_{r}$. Since the common method and the proofs of the statements for type $B_{r}$ were described in [IIKKN] in detail, in this paper, we skip the proofs of most statements, and concentrate on presenting the results with emphasis on the special features of each case. Notably, the tropical Y-system at level 2, which is the core part in the entire method, is quite specific to each case.

While we try to make the paper as self-contained as possible, we also try to minimize duplication with [IIKKN]. Therefore, we have to ask the reader for tolerating numerous references to the companion paper [IIKKN] for the things which are omitted. In particular, basic definitions for cluster algebras are summarized in [IIKKN, Section 2.1].

The organization of the paper is as follows. In Section 2 we present the main results as well as the T-systems and Y-systems for each type. In Section 3 the results for type $C_{r}$ are established. The key tropical Y-system at level 2 is described in detail in Section 3.6. In Section 4 the results for type $F_{4}$ are proved. In Section 5 we give the results for type $G_{2}$. In Section 6 we list the known mutation equivalences of quivers corresponding to the T-systems and Y-systems.

\section{$\S 2$. Main results}

\section{§2.1. Restricted T-systems and $\mathrm{Y}$-systems of types $C_{r}, F_{4}$, and $G_{2}$}

Let $X_{r}$ be the Dynkin diagram of type $C_{r}, F_{4}$, or $G_{2}$ with $\operatorname{rank} r$, and $I=\{1, \ldots, r\}$ be the enumeration of the vertices of $X_{r}$ :
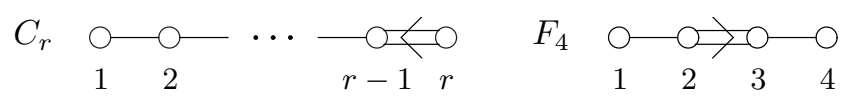

$$
G_{2} \Longleftrightarrow 0
$$

Let $h$ and $h^{\vee}$ be the Coxeter number and the dual Coxeter number of $X_{r}$, respectively. Then 


\begin{tabular}{c|ccc}
\hline$X_{r}$ & $C_{r}$ & $F_{4}$ & $G_{2}$ \\
\hline$h$ & $2 r$ & 12 & 6 \\
$h^{\vee}$ & $r+1$ & 9 & 4 \\
\hline
\end{tabular}

We define numbers $t$ and $t_{a}(a \in I)$ by

$$
t=\left\{\begin{array}{ll}
2, & X_{r}=C_{r}, F_{4}, \\
3, & X_{r}=G_{2},
\end{array} \quad t_{a}= \begin{cases}1, & \alpha_{a} \text { long root }, \\
t, & \alpha_{a} \text { short root. }\end{cases}\right.
$$

For a given integer $\ell \geq 2$, we introduce a set of triplets

$$
\mathcal{I}_{\ell}=\mathcal{I}_{\ell}\left(X_{r}\right):=\left\{(a, m, u) \mid a \in I ; m=1, \ldots, t_{a} \ell-1 ; u \in(1 / t) \mathbb{Z}\right\} .
$$

Definition 2.1 ([KNS]). Fix an integer $\ell \geq 2$. The level $\ell$ restricted $T$-system $\mathbb{T}_{\ell}\left(X_{r}\right)$ of type $X_{r}$ (with the unit boundary condition) is the following system of relations for a family of variables $T_{\ell}=\left\{T_{m}^{(a)}(u) \mid(a, m, u) \in \mathcal{I}_{\ell}\right\}$, where $T_{m}^{(0)}(u)=$ $T_{0}^{(a)}(u)=1$, and furthermore, $T_{t_{a} \ell}^{(a)}(u)=1$ (the unit boundary condition) if they occur in the right hand sides of the relations.

(Here and throughout the paper, $2 m$ (resp. $2 m+1$ ) on the left hand sides, for example, represents elements $2,4, \ldots$ (resp. $1,3, \ldots)$.)

For $X_{r}=C_{r}$,

$$
\begin{aligned}
T_{m}^{(a)}\left(u-\frac{1}{2}\right) T_{m}^{(a)}\left(u+\frac{1}{2}\right)= & T_{m-1}^{(a)}(u) T_{m+1}^{(a)}(u)+T_{m}^{(a-1)}(u) T_{m}^{(a+1)}(u) \\
T_{2 m}^{(r-1)}\left(u-\frac{1}{2}\right) T_{2 m}^{(r-1)}\left(u+\frac{1}{2}\right)= & T_{2 m-1}^{(r-1)}(u) T_{2 m-1}^{(r-1)}(u) \\
& +T_{2 m}^{(r-2)}(u) T_{m}^{(r)}\left(u-\frac{1}{2}\right) T_{m}^{(r)}\left(u+\frac{1}{2}\right), \\
T_{2 m+1}^{(r-1)}\left(u-\frac{1}{2}\right) T_{2 m+1}^{(r-1)}\left(u+\frac{1}{2}\right)= & T_{2 m}^{(r-1)}(u) T_{2 m+2}^{(r-1)}(u) \\
& +T_{2 m+1}^{(r-2)}(u) T_{m}^{(r)}(u) T_{m+1}^{(r)}(u), \\
T_{m}^{(r)}(u-1) T_{m}^{(r)}(u+1)= & T_{m-1}^{(r)}(u) T_{m+1}^{(r)}(u)+T_{2 m}^{(r-1)}(u) .
\end{aligned}
$$

For $X_{r}=F_{4}$,

$$
\begin{aligned}
T_{m}^{(1)}(u-1) T_{m}^{(1)}(u+1)= & T_{m-1}^{(1)}(u) T_{m+1}^{(1)}(u)+T_{m}^{(2)}(u), \\
T_{m}^{(2)}(u-1) T_{m}^{(2)}(u+1)= & T_{m-1}^{(2)}(u) T_{m+1}^{(2)}(u)+T_{m}^{(1)}(u) T_{2 m}^{(3)}(u), \\
T_{2 m}^{(3)}\left(u-\frac{1}{2}\right) T_{2 m}^{(3)}\left(u+\frac{1}{2}\right)= & T_{2 m-1}^{(3)}(u) T_{2 m+1}^{(3)}(u) \\
& +T_{m}^{(2)}\left(u-\frac{1}{2}\right) T_{m}^{(2)}\left(u+\frac{1}{2}\right) T_{2 m}^{(4)}(u), \\
T_{2 m+1}^{(3)}\left(u-\frac{1}{2}\right) T_{2 m+1}^{(3)}\left(u+\frac{1}{2}\right)= & T_{2 m}^{(3)}(u) T_{2 m+2}^{(3)}(u)+T_{m}^{(2)}(u) T_{m+1}^{(2)}(u) T_{2 m+1}^{(4)}(u), \\
T_{m}^{(4)}\left(u-\frac{1}{2}\right) T_{m}^{(4)}\left(u+\frac{1}{2}\right)= & T_{m-1}^{(4)}(u) T_{m+1}^{(4)}(u)+T_{m}^{(3)}(u) .
\end{aligned}
$$


For $X_{r}=G_{2}$,

$$
\begin{aligned}
T_{m}^{(1)}(u-1) T_{m}^{(1)}(u+1)= & T_{m-1}^{(1)}(u) T_{m+1}^{(1)}(u)+T_{3 m}^{(2)}(u), \\
T_{3 m}^{(2)}\left(u-\frac{1}{3}\right) T_{3 m}^{(2)}\left(u+\frac{1}{3}\right)= & T_{3 m-1}^{(2)}(u) T_{3 m+1}^{(2)}(u) \\
& +T_{m}^{(1)}\left(u-\frac{2}{3}\right) T_{m}^{(1)}(u) T_{m}^{(1)}\left(u+\frac{2}{3}\right), \\
T_{3 m+1}^{(2)}\left(u-\frac{1}{3}\right) T_{3 m+1}^{(2)}\left(u+\frac{1}{3}\right)= & T_{3 m}^{(2)}(u) T_{3 m+2}^{(2)}(u) \\
& +T_{m}^{(1)}\left(u-\frac{1}{3}\right) T_{m}^{(1)}\left(u+\frac{1}{3}\right) T_{m+1}^{(1)}(u), \\
T_{3 m+2}^{(2)}\left(u-\frac{1}{3}\right) T_{3 m+2}^{(2)}\left(u+\frac{1}{3}\right)= & T_{3 m+1}^{(2)}(u) T_{3 m+3}^{(2)}(u) \\
& +T_{m}^{(1)}(u) T_{m+1}^{(1)}\left(u-\frac{1}{3}\right) T_{m+1}^{(1)}\left(u+\frac{1}{3}\right) .
\end{aligned}
$$

Definition $2.2([\mathrm{KN}])$. Fix an integer $\ell \geq 2$. The level $\ell$ restricted $Y$-system $\mathbb{Y}_{\ell}\left(X_{r}\right)$ of type $X_{r}$ is the following system of relations for a family of variables $Y_{\ell}=\left\{Y_{m}^{(a)}(u) \mid(a, m, u) \in \mathcal{I}_{\ell}\right\}$, where $Y_{m}^{(0)}(u)=Y_{0}^{(a)}(u)^{-1}=Y_{t_{a} \ell}^{(a)}(u)^{-1}=0$ if they occur in the right hand sides in the relations:

For $X_{r}=C_{r}$,

$$
\begin{aligned}
Y_{m}^{(a)}\left(u-\frac{1}{2}\right) Y_{m}^{(a)}\left(u+\frac{1}{2}\right)= & \frac{\left(1+Y_{m}^{(a-1)}(u)\right)\left(1+Y_{m}^{(a+1)}(u)\right)}{\left(1+Y_{m-1}^{(a)}(u)^{-1}\right)\left(1+Y_{m+1}^{(a)}(u)^{-1}\right)}(1 \leq a \leq r-2), \\
Y_{2 m}^{(r-1)}\left(u-\frac{1}{2}\right) Y_{2 m}^{(r-1)}\left(u+\frac{1}{2}\right)= & \frac{\left(1+Y_{2 m}^{(r-2)}(u)\right)\left(1+Y_{m}^{(r)}(u)\right)}{\left(1+Y_{2 m-1}^{(r-1)}(u)^{-1}\right)\left(1+Y_{2 m+1}^{(r-1)}(u)^{-1}\right)}, \\
Y_{2 m+1}^{(r-1)}\left(u-\frac{1}{2}\right) Y_{2 m+1}^{(r-1)}\left(u+\frac{1}{2}\right)= & \frac{1+Y_{2 m+1}^{(r-2)}(u)}{\left(1+Y_{2 m}^{(r-1)}(u)^{-1}\right)\left(1+Y_{2 m+2}^{(r-1)}(u)^{-1}\right)}, \\
& \left(1+Y_{2 m+1}^{(r-1)}(u)\right)\left(1+Y_{2 m-1}^{(r-1)}(u)\right) \\
Y_{m}^{(r)}(u-1) Y_{m}^{(r)}(u+1)= & \frac{\times\left(1+Y_{2 m}^{(r-1)}\left(u-\frac{1}{2}\right)\right)\left(1+Y_{2 m}^{(r-1)}\left(u+\frac{1}{2}\right)\right)}{\left(1+Y_{m-1}^{(r)}(u)^{-1}\right)\left(1+Y_{m+1}^{(r)}(u)^{-1}\right)} .
\end{aligned}
$$

For $X_{r}=F_{4}$,

$$
\begin{aligned}
Y_{m}^{(1)}(u-1) Y_{m}^{(1)}(u+1)= & \frac{1+Y_{m}^{(2)}(u)}{\left(1+Y_{m-1}^{(1)}(u)^{-1}\right)\left(1+Y_{m+1}^{(1)}(u)^{-1}\right)}, \\
Y_{m}^{(2)}(u-1) Y_{m}^{(2)}(u+1)= & \frac{\left(1+Y_{m}^{(1)}(u)\right)\left(1+Y_{2 m-1}^{(3)}(u)\right)\left(1+Y_{2 m+1}^{(3)}(u)\right)}{\left(1+Y_{m-1}^{(2)}\left(u-\frac{1}{2}\right)\right)\left(1+Y_{2 m}^{(3)}\left(u+\frac{1}{2}\right)\right)} \\
Y_{2 m}^{(3)}\left(u-\frac{1}{2}\right) Y_{2 m}^{(3)}\left(u+\frac{1}{2}\right)= & \frac{\left(1+Y_{m}^{(2)}(u)\right)\left(1+Y_{2 m}^{(2)}(u)\right)}{\left(1+Y_{2 m-1}^{(3)}(u)^{-1}\right)\left(1+Y_{2 m+1}^{(3)}(u)^{-1}\right)},
\end{aligned}
$$




$$
\begin{aligned}
Y_{2 m+1}^{(3)}\left(u-\frac{1}{2}\right) Y_{2 m+1}^{(3)}\left(u+\frac{1}{2}\right) & =\frac{1+Y_{2 m+1}^{(4)}(u)}{\left(1+Y_{2 m}^{(3)}(u)^{-1}\right)\left(1+Y_{2 m+2}^{(3)}(u)^{-1}\right)} \\
Y_{m}^{(4)}\left(u-\frac{1}{2}\right) Y_{m}^{(4)}\left(u+\frac{1}{2}\right) & =\frac{1+Y_{m}^{(3)}(u)}{\left(1+Y_{m-1}^{(4)}(u)^{-1}\right)\left(1+Y_{m+1}^{(4)}(u)^{-1}\right)}
\end{aligned}
$$

For $X_{r}=G_{2}$,

$$
\begin{aligned}
&\left(1+Y_{3 m-2}^{(2)}(u)\right)\left(1+Y_{3 m+2}^{(2)}(u)\right) \\
& \times\left(1+Y_{3 m-1}^{(2)}\left(u-\frac{1}{3}\right)\right)\left(1+Y_{3 m-1}^{(2)}\left(u+\frac{1}{3}\right)\right) \\
& \times\left(1+Y_{3 m+1}^{(2)}\left(u-\frac{1}{3}\right)\right)\left(1+Y_{3 m+1}^{(2)}\left(u+\frac{1}{3}\right)\right) \\
& \times\left(1+Y_{3 m}^{(2)}\left(u-\frac{2}{3}\right)\right)\left(1+Y_{3 m}^{(2)}\left(u+\frac{2}{3}\right)\right) \\
& Y_{m}^{(1)}(u-1) Y_{m}^{(1)}(u+1)=\frac{\times\left(1+Y_{3 m}^{(2)}(u)\right)}{\left(1+Y_{m-1}^{(1)}(u)^{-1}\right)\left(1+Y_{m+1}^{(1)}(u)^{-1}\right)} \\
& Y_{3 m}^{(2)}\left(u-\frac{1}{3}\right) Y_{3 m}^{(2)}\left(u+\frac{1}{3}\right)= \\
& Y_{3 m+1}^{(2)}\left(u-\frac{1}{3}\right) Y_{3 m+1}^{(2)}\left(u+\frac{1}{3}\right)= \\
& Y_{3 m+2}^{(2)}\left(u-\frac{1}{3}\right) Y_{3 m+2}^{(2)}\left(u+\frac{1}{3}\right)= \frac{1+Y_{m}^{(1)}(u)}{\left(1+Y_{3 m}^{(2)}(u)^{-1}\right)\left(1+Y_{3 m+2}^{(2)}(u)^{-1}\right)}, \\
&\left(1+Y_{3 m+1}^{(2)}(u)^{-1}\right)\left(1+Y_{3 m+3}^{(2)}(u)^{-1}\right)
\end{aligned}
$$

Let us write (2.4)-(2.6) in a unified manner

$$
\begin{aligned}
T_{m}^{(a)}\left(u-\frac{1}{t_{a}}\right) T_{m}^{(a)} & \left(u+\frac{1}{t_{a}}\right) \\
& =T_{m-1}^{(a)}(u) T_{m+1}^{(a)}(u)+\prod_{(b, k, v) \in \mathcal{I}_{\ell}} T_{k}^{(b)}(v)^{G(b, k, v ; a, m, u)} .
\end{aligned}
$$

Define the transposition ${ }^{t} G(b, k, v ; a, m, u)=G(a, m, u ; b, k, v)$. Then $(2.7)-(2.9)$ can be written as



\section{$\S 2.2$. Periodicities}

Definition 2.3. Let $\mathcal{T}_{\ell}\left(X_{r}\right)$ be the commutative ring over $\mathbb{Z}$ with identity element, with generators $T_{m}^{(a)}(u)^{ \pm 1}\left((a, m, u) \in \mathcal{I}_{\ell}\right)$ and relations $\mathbb{T}_{\ell}\left(X_{r}\right)$ together with $T_{m}^{(a)}(u) T_{m}^{(a)}(u)^{-1}=1$. Let $\mathcal{T}_{\ell}^{\circ}\left(X_{r}\right)$ be the subring of $\mathcal{T}_{\ell}\left(X_{r}\right)$ generated by $T_{m}^{(a)}(u)\left((a, m, u) \in \mathcal{I}_{\ell}\right)$.

Definition 2.4. Let $y_{\ell}\left(X_{r}\right)$ be the semifield with generators $Y_{m}^{(a)}(u)((a, m, u)$ $\in \mathcal{I}_{\ell}$ and relations $\mathbb{Y}_{\ell}\left(X_{r}\right)$. Let $y_{\ell}^{\circ}\left(X_{r}\right)$ be the multiplicative subgroup of $y_{\ell}\left(X_{r}\right)$ 
generated by $Y_{m}^{(a)}(u), 1+Y_{m}^{(a)}(u)\left((a, m, u) \in \mathcal{I}_{\ell}\right)$. (Here we use the symbol + instead of $\oplus$ for simplicity.)

The first main result of the paper concern the periodicities of the T-systems and Y-systems.

Theorem 2.5 (Conjectured in [IIKNS]). The following relations hold in $\mathcal{T}_{\ell}^{\circ}\left(X_{r}\right)$ :

(i) Half periodicity: $T_{m}^{(a)}\left(u+h^{\vee}+\ell\right)=T_{t_{a} \ell-m}^{(a)}(u)$.

(ii) Full periodicity: $T_{m}^{(a)}\left(u+2\left(h^{\vee}+\ell\right)\right)=T_{m}^{(a)}(u)$.

Theorem 2.6 (Conjectured in $[\mathrm{KNS}]$ ). The following relations hold in $y_{\ell}^{\circ}\left(X_{r}\right)$ :

(i) Half periodicity: $Y_{m}^{(a)}\left(u+h^{\vee}+\ell\right)=Y_{t_{a} \ell-m}^{(a)}(u)$.

(ii) Full periodicity: $Y_{m}^{(a)}\left(u+2\left(h^{\vee}+\ell\right)\right)=Y_{m}^{(a)}(u)$.

\section{§2.3. Dilogarithm identities}

Let $L(x)$ be the Rogers dilogarithm function

$$
L(x)=-\frac{1}{2} \int_{0}^{x}\left\{\frac{\log (1-y)}{y}+\frac{\log y}{1-y}\right\} d y \quad(0 \leq x \leq 1) .
$$

We introduce the constant version of the Y-system.

Definition 2.7. Fix an integer $\ell \geq 2$. The level $\ell$ restricted constant $Y$-system $\mathbb{Y}_{\ell}^{\mathrm{c}}\left(X_{r}\right)$ of type $X_{r}$ is the following system of relations for a family of variables $Y_{\ell}^{\mathrm{c}}=\left\{Y_{m}^{(a)} \mid a \in I ; m=1, \ldots, t_{a} \ell-1\right\}$, where $Y_{m}^{(0)}=Y_{0}^{(a)-1}=Y_{t_{a} \ell}^{(a)-1}=0$ if they occur on the right hand sides of the relations.

For $X_{r}=C_{r}$,

$$
\begin{aligned}
\left(Y_{m}^{(a)}\right)^{2} & =\frac{\left(1+Y_{m}^{(a-1)}\right)\left(1+Y_{m}^{(a+1)}\right)}{\left(1+Y_{m-1}^{(a)}-1\right)\left(1+Y_{m+1}^{(a)}-1\right)} \quad(1 \leq a \leq r-2), \\
\left(Y_{2 m}^{(r-1)}\right)^{2} & =\frac{\left(1+Y_{2 m}^{(r-2)}\right)\left(1+Y_{m}^{(r)}\right)}{\left(1+Y_{2 m-1}^{(r-1)}-1\right)\left(1+Y_{2 m+1}^{(r-1)}-1\right)}, \\
\left(Y_{2 m+1}^{(r-1)}\right)^{2} & =\frac{1+Y_{2 m+1}^{(r-2)}}{\left(1+Y_{2 m}^{(r-1)}-1\right)\left(1+Y_{2 m+2}^{(r-1)}-1\right)}, \\
\left(Y_{m}^{(r)}\right)^{2} & =\frac{\left(1+Y_{2 m-1}^{(r-1)}\right)\left(1+Y_{2 m}^{(r-1)}\right)^{2}\left(1+Y_{2 m+1}^{(r-1)}\right)}{\left(1+Y_{m-1}^{(r)}-1\right)\left(1+Y_{m+1}^{(r)}-1\right)} .
\end{aligned}
$$


For $X_{r}=F_{4}$,

$$
\begin{aligned}
\left(Y_{m}^{(1)}\right)^{2} & =\frac{1+Y_{m}^{(2)}}{\left(1+Y_{m-1}^{(1)}-1\right)\left(1+Y_{m+1}^{(1)}-1\right)} \\
\left(Y_{m}^{(2)}\right)^{2} & =\frac{\left(1+Y_{m}^{(1)}\right)\left(1+Y_{2 m-1}^{(3)}\right)\left(1+Y_{2 m}^{(3)}\right)^{2}\left(1+Y_{2 m+1}^{(3)}\right)}{\left(1+Y_{m-1}^{(2)}-1\right)\left(1+Y_{m+1}^{(2)}-1\right)} \\
\left(Y_{2 m}^{(3)}\right)^{2} & =\frac{\left(1+Y_{m}^{(2)}\right)\left(1+Y_{2 m}^{(4)}\right)}{\left(1+Y_{2 m-1}^{(3)}-1\right)\left(1+Y_{2 m+1}^{(3)}-1\right)} \\
\left(Y_{2 m+1}^{(3)}\right)^{2} & =\frac{1+Y_{2 m+1}^{(4)}}{\left(1+Y_{2 m}^{(3)}-1\right)\left(1+Y_{2 m+2}^{(3)}-1\right)} \\
\left(Y_{m}^{(4)}\right)^{2} & =\frac{1+Y_{m}^{(3)}}{\left(1+Y_{m-1}^{(4)}-1\right)\left(1+Y_{m+1}^{(4)}-1\right)}
\end{aligned}
$$

For $X_{r}=G_{2}$,

$$
\begin{aligned}
\left(Y_{m}^{(1)}\right)^{2} & =\frac{\left(1+Y_{3 m-2}^{(2)}\right)\left(1+Y_{3 m-1}^{(2)}\right)^{2}\left(1+Y_{3 m}^{(2)}\right)^{3}\left(1+Y_{3 m+1}^{(2)}\right)^{2}\left(1+Y_{3 m+2}^{(2)}\right)}{\left(1+Y_{m-1}^{(1)}-1\right)\left(1+Y_{m+1}^{(1)}-1\right)} \\
\left(Y_{3 m}^{(2)}\right)^{2} & =\frac{1+Y_{m}^{(1)}}{\left(1+Y_{3 m-1}^{(2)}-1\right)\left(1+Y_{3 m+1}^{(2)}-1\right)}, \\
\left(Y_{3 m+1}^{(2)}\right)^{2} & =\frac{1}{\left(1+Y_{3 m}^{(2)}-1\right)\left(1+Y_{3 m+2}^{(2)}-1\right)}, \\
\left(Y_{3 m+2}^{(2)}\right)^{2} & =\frac{1}{\left(1+Y_{3 m+1}^{(2)}-1\right)\left(1+Y_{3 m+3}^{(2)}-1\right)} .
\end{aligned}
$$

Proposition 2.8. There exists a unique positive real solution of $\mathbb{Y}_{\ell}^{\mathrm{c}}\left(X_{r}\right)$.

Proof. The proof of [IIKKN, Proposition 1.8] is applicable.

The second main result of the paper is the dilogarithm identities conjectured by Kirillov [Ki, Eq. (7)], and properly corrected by Kuniba [Ku, Eqs. (A.1a), (A.1c)].

Theorem 2.9 (Dilogarithm identities). Suppose that a family of positive real numbers $\left\{Y_{m}^{(a)} \mid a \in I ; m=1, \ldots, t_{a} \ell-1\right\}$ satisfies $\mathbb{Y}_{\ell}^{\mathrm{c}}\left(X_{r}\right)$. Then

$$
\frac{6}{\pi^{2}} \sum_{a \in I} \sum_{m=1}^{t_{a} \ell-1} L\left(\frac{Y_{m}^{(a)}}{1+Y_{m}^{(a)}}\right)=\frac{\ell \operatorname{dim} \mathfrak{g}}{h^{\vee}+\ell}-r
$$

where $\mathfrak{g}$ is the simple Lie algebra of type $X_{r}$. 
The right hand side of $(2.16)$ is equal to

$$
\frac{r\left(\ell h-h^{\vee}\right)}{h^{\vee}+\ell} .
$$

In fact, we prove a functional generalization of Theorem 2.9.

Theorem 2.10 (Functional dilogarithm identities). Suppose that a family of positive real numbers $\left\{Y_{m}^{(a)}(u) \mid(a, m, u) \in \mathcal{I}_{\ell}\right\}$ satisfies $\mathbb{Y}_{\ell}\left(X_{r}\right)$. Then

$$
\begin{aligned}
\frac{6}{\pi^{2}} \sum_{\substack{(a, m, u) \in \mathcal{I}_{\ell} \\
0 \leq u<2\left(h^{\vee}+\ell\right)}} L\left(\frac{Y_{m}^{(a)}(u)}{1+Y_{m}^{(a)}(u)}\right) & =2 \operatorname{tr}\left(\ell h-h^{\vee}\right) \\
& = \begin{cases}4 r(2 r \ell-r-1), & C_{r}, \\
48(4 \ell-3), & F_{4}, \\
24(3 \ell-2), & G_{2},\end{cases} \\
\frac{6}{\pi^{2}} \sum_{\substack{(a, m, u) \in \mathcal{I}_{\ell} \\
0 \leq u<2\left(h^{\vee}+\ell\right)}} L\left(\frac{1}{1+Y_{m}^{(a)}(u)}\right) & = \begin{cases}4 \ell(2 r \ell-\ell-1), & C_{r}, \\
8 \ell(3 \ell+1), & F_{4}, \\
12 \ell(2 \ell+1), & G_{2} .\end{cases}
\end{aligned}
$$

The two identities (2.18) and (2.19) are equivalent to each other, since the sum of the right hand sides is equal to $2 t\left(h^{\vee}+\ell\right)\left(\left(\sum_{a \in I} t_{a}\right) \ell-r\right)$, which is the total number of $(a, m, u) \in \mathcal{I}_{\ell}$ in the region $0 \leq u<2\left(h^{\vee}+\ell\right)$.

It is clear that Theorem 2.9 follows from Theorem 2.10 .

\section{$\S 3$. Type $C_{r}$}

The $C_{r}$ case is quite parallel to the $B_{r}$ case. For the reader's convenience, we repeat most of the basic definitions and results from [IIKKN]. Most propositions are proved in a parallel manner to the $B_{r}$ case, so that proofs are omitted. The properties of the tropical Y-system at level 2 (Proposition 3.10) are crucial and specific to $C_{r}$. Since its derivation is a little more complicated than in the $B_{r}$ case, an outline of the proof is provided.

\section{§3.1. Parity decompositions of T-systems and Y-systems}

For a triplet $(a, m, u) \in \mathcal{I}_{\ell}$, we define the 'parity conditions' $\mathbf{P}_{+}$and $\mathbf{P}_{-}$by

$$
\begin{aligned}
& \mathbf{P}_{+}: r+a+m+2 u \text { is odd if } a \neq r ; 2 u \text { is even if } a=r \\
& \mathbf{P}_{-}: r+a+m+2 u \text { is even if } a \neq r ; 2 u \text { is odd if } a=r .
\end{aligned}
$$



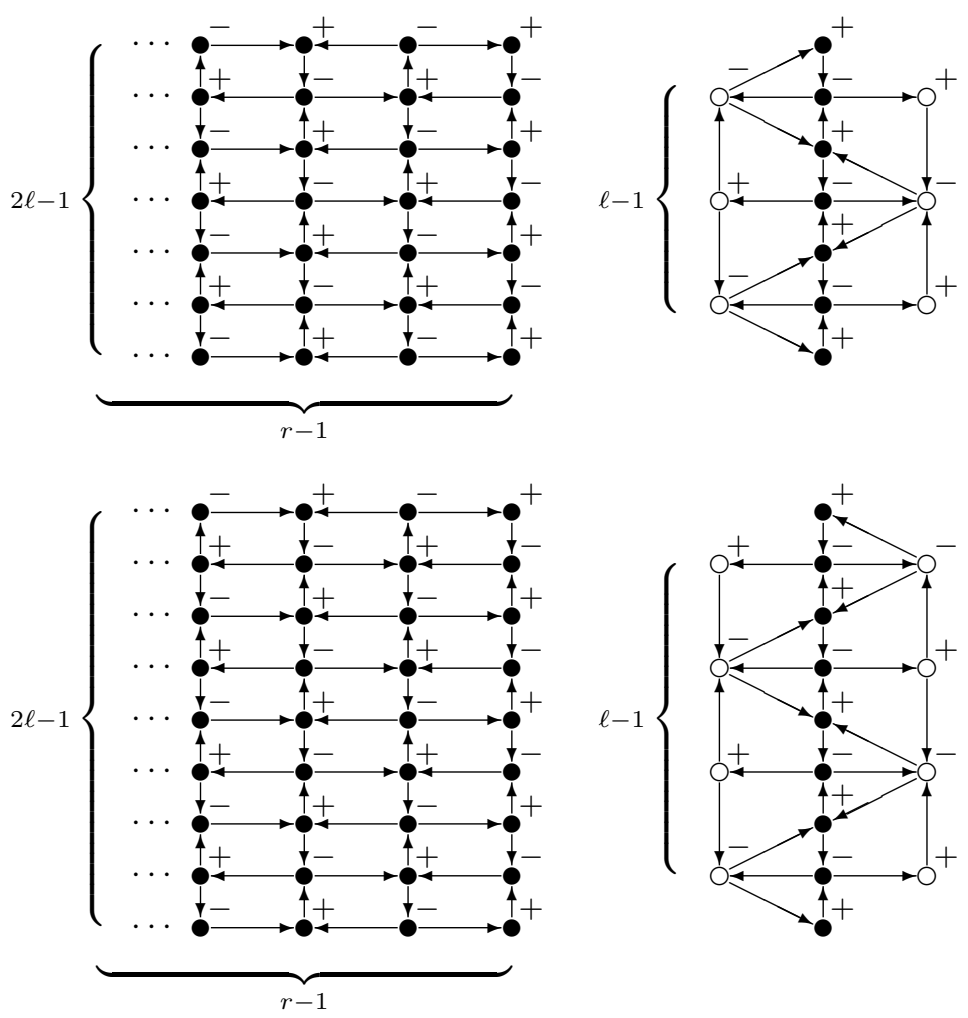

Figure 1. The quiver $Q_{\ell}\left(C_{r}\right)$ for $\ell$ even (top) and for $\ell$ odd (botom), where we identify the rightmost column in the left quiver with the middle column in the right quiver.

We write, for example, $(a, m, u): \mathbf{P}_{+}$if $(a, m, u)$ satisfies $\mathbf{P}_{+}$. We have $\mathcal{I}_{\ell}=$ $\mathcal{I}_{\ell+} \sqcup \mathcal{I}_{\ell-}$, where $\mathcal{I}_{\ell \varepsilon}$ is the set of all $(a, m, u): \mathbf{P}_{\varepsilon}$.

Define $\mathcal{T}_{\ell}^{\circ}\left(C_{r}\right)_{\varepsilon}(\varepsilon= \pm)$ to be the subring of $\mathcal{T}_{\ell}^{\circ}\left(C_{r}\right)$ generated by $T_{m}^{(a)}(u)$ $\left((a, m, u) \in \mathcal{I}_{\ell \varepsilon}\right)$. Then we have $\mathcal{T}_{\ell}^{\circ}\left(C_{r}\right)_{+} \simeq \mathcal{T}_{\ell}^{\circ}\left(C_{r}\right)_{-}$via $T_{m}^{(a)}(u) \mapsto T_{m}^{(a)}\left(u+\frac{1}{2}\right)$ and

$$
\mathcal{T}_{\ell}^{\circ}\left(C_{r}\right) \simeq \mathcal{T}_{\ell}^{\circ}\left(C_{r}\right)_{+} \otimes_{\mathbb{Z}} \mathcal{T}_{\ell}^{\circ}\left(C_{r}\right)_{-}
$$

For a triplet $(a, m, u) \in \mathcal{I}_{\ell}$, we set other 'parity conditions' $\mathbf{P}_{+}^{\prime}$ and $\mathbf{P}_{-}^{\prime}$ by

$$
\begin{aligned}
& \mathbf{P}_{+}^{\prime}: r+a+m+2 u \text { is even if } a \neq r ; 2 u \text { is even if } a=r, \\
& \mathbf{P}_{-}^{\prime}: r+a+m+2 u \text { is odd if } a \neq r ; 2 u \text { is odd if } a=r .
\end{aligned}
$$


R. INOUE ET AL.

We have $\mathcal{I}_{\ell}=\mathcal{I}_{\ell+}^{\prime} \sqcup \mathcal{I}_{\ell-}^{\prime}$, where $\mathcal{I}_{\ell \varepsilon}^{\prime}$ is the set of all $(a, m, u): \mathbf{P}_{\varepsilon}^{\prime}$. We also have

$$
(a, m, u): \mathbf{P}_{+}^{\prime} \Leftrightarrow\left(a, m, u \pm \frac{1}{t_{a}}\right): \mathbf{P}_{+} .
$$

Define $y_{\ell}^{\circ}\left(C_{r}\right)_{\varepsilon}(\varepsilon= \pm)$ to be the subgroup of $y_{\ell}^{\circ}\left(C_{r}\right)$ generated by $Y_{m}^{(a)}(u)$, $1+Y_{m}^{(a)}(u)\left((a, m, u) \in \mathcal{I}_{\ell \varepsilon}^{\prime}\right)$. Then $y_{\ell}^{\circ}\left(C_{r}\right)_{+} \simeq y_{\ell}^{\circ}\left(C_{r}\right)_{-}$via $Y_{m}^{(a)}(u) \mapsto Y_{m}^{(a)}\left(u+\frac{1}{2}\right)$, $1+Y_{m}^{(a)}(u) \mapsto 1+Y_{m}^{(a)}\left(u+\frac{1}{2}\right)$, and

$$
y_{\ell}^{\circ}\left(C_{r}\right) \simeq y_{\ell}^{\circ}\left(C_{r}\right)_{+} \times y_{\ell}^{\circ}\left(C_{r}\right)_{-} .
$$

\section{$\S 3.2$. Quiver $Q_{\ell}\left(C_{r}\right)$}

With type $C_{r}$ and $\ell \geq 2$ we associate the quiver $Q_{\ell}\left(C_{r}\right)$ by Figure 1 , where the rightmost column in the left quiver and the middle column in the right quiver are identified. Also, we assign an empty or filled circle $\circ / \bullet$ and a sign $+/-$ to each vertex.

Let us choose the index set $\mathbf{I}$ of the vertices of $Q_{\ell}\left(C_{r}\right)$ so that $\mathbf{i}=\left(i, i^{\prime}\right) \in \mathbf{I}$ represents the vertex in the $i^{\prime}$ th row (from the bottom) and the $i$ th column (from the left) of the left quiver for $i=1, \ldots, r-1$, in the right column of the right quiver for $i=r$, and in the left column of the right quiver for $i=r+1$. Thus, $i=1, \ldots, r+1$, and $i^{\prime}=1, \ldots, \ell-1$ if $i \neq r, r+1$, while $i^{\prime}=1, \ldots, 2 \ell-1$ if $i=r, r+1$. We use the natural notation $\mathbf{I}^{\circ}\left(\operatorname{resp} . \mathbf{I}_{+}^{\circ}\right)$ for the set of vertices $\mathbf{i}$ with property $\circ$ (resp. $\circ$ and + ), and so on. We have $\mathbf{I}=\mathbf{I}^{\circ} \sqcup \mathbf{I}^{\bullet}=\mathbf{I}_{+}^{\circ} \sqcup \mathbf{I}_{-}^{\circ} \sqcup \mathbf{I}_{+}^{\bullet} \sqcup \mathbf{I}_{-}^{\bullet}$.

We define composite mutations,

$$
\mu_{+}^{\circ}=\prod_{\mathbf{i} \in \mathbf{I}_{+}^{\circ}} \mu_{\mathbf{i}}, \quad \mu_{-}^{\circ}=\prod_{\mathbf{i} \in \mathbf{I}_{-}^{\circ}} \mu_{\mathbf{i}}, \quad \mu_{+}^{\bullet}=\prod_{\mathbf{i} \in \mathbf{I}_{+}^{\bullet}} \mu_{\mathbf{i}}, \quad \mu_{-}^{\bullet}=\prod_{\mathbf{i} \in \mathbf{I}_{-}^{\bullet}} \mu_{\mathbf{i}} .
$$

Note that they do not depend on the order of the product.

Let $\boldsymbol{r}$ be the involution acting on $\mathbf{I}$ by left-right reflection of the right quiver. Let $\boldsymbol{\omega}$ be the involution acting on $\mathbf{I}$ defined by, for even $r$, up-down reflection of the left quiver and $180^{\circ}$ rotation of the right quiver; and for odd $r$, up-down reflection of the left and right quivers. Let $\boldsymbol{r}\left(Q_{\ell}\left(C_{r}\right)\right)$ and $\boldsymbol{\omega}\left(Q_{\ell}\left(C_{r}\right)\right)$ denote the quivers induced from $Q_{\ell}\left(C_{r}\right)$ by $\boldsymbol{r}$ and $\boldsymbol{\omega}$, respectively. For example, if there is an arrow $\mathbf{i} \rightarrow \mathbf{j}$ in $Q_{\ell}\left(C_{r}\right)$, then there is an arrow $\boldsymbol{r}(\mathbf{i}) \rightarrow \boldsymbol{r}(\mathbf{j})$ in $\boldsymbol{r}\left(Q_{\ell}\left(C_{r}\right)\right)$. For a quiver $Q$, $Q^{\text {op }}$ denotes the opposite quiver.

Lemma 3.1. Let $Q=Q_{\ell}\left(C_{r}\right)$.

(i) We have a periodic sequence of mutations of quivers

$$
Q \stackrel{\mu_{+}^{\bullet} \mu_{+}^{\circ}}{\longleftrightarrow} Q^{\text {op }} \stackrel{\mu_{-}^{\bullet}}{\longleftrightarrow} \boldsymbol{r}(Q) \stackrel{\mu_{+}^{\bullet} \mu_{-}^{\circ}}{\longleftrightarrow} \boldsymbol{r}(Q)^{\mathrm{op}} \stackrel{\mu_{-}^{\bullet}}{\longleftrightarrow} Q .
$$

(ii) $\boldsymbol{\omega}(Q)=Q$ if $h^{\vee}+\ell$ is even, and $\boldsymbol{\omega}(Q)=\boldsymbol{r}(Q)$ if $h^{\vee}+\ell$ is odd. 


\section{$\S 3.3$. Cluster algebra and alternative labels}

It is standard to identify a quiver $Q$ with no loop and no 2-cycle with a skewsymmetric matrix $B$. We use the following convention for the direction of arrows:

$$
i \rightarrow j \Leftrightarrow B_{i j}=1 \text {. }
$$

(In this paper we only encounter the situation where $B_{i j}=-1,0,1$.) Let $B_{\ell}\left(C_{r}\right)$ be the skew-symmetric matrix corresponding to the quiver $Q_{\ell}\left(C_{r}\right)$. In the rest of the section, we set $B=\left(B_{\mathbf{i j}}\right)_{\mathbf{i}, \mathbf{j} \in \mathbf{I}}=B_{\ell}\left(C_{r}\right)$ unless otherwise mentioned.

Let $\mathcal{A}(B, x, y)$ be the cluster algebra with coefficients in the universal semifield $\mathbb{Q}_{\mathrm{sf}}(y)$, where $(B, x, y)$ is the initial seed [FZ2]. See also [IIKKN, Section 2.1] for the conventions and notations on cluster algebras we employ. (Here we use the symbol + instead of $\oplus$ in $\mathbb{Q}_{\text {sf }}(y)$, since it is the ordinary addition of subtractionfree expressions of rational functions of $y$.)

Definition 3.2. The coefficient group $\mathcal{G}(B, y)$ associated with $\mathcal{A}(B, x, y)$ is the multiplicative subgroup of the semifield $\mathbb{Q}_{\mathrm{sf}}(y)$ generated by all the coefficients $y_{\mathbf{i}}^{\prime}$ of $\mathcal{A}(B, x, y)$ together with $1+y_{\mathbf{i}}^{\prime}$.

In view of Lemma 3.1 we set $x(0)=x, y(0)=y$ and define clusters $x(u)=$ $\left(x_{\mathbf{i}}(u)\right)_{\mathbf{i} \in \mathbf{I}}\left(u \in \frac{1}{2} \mathbb{Z}\right)$ and coefficient tuples $y(u)=\left(y_{\mathbf{i}}(u)\right)_{\mathbf{i} \in \mathbf{I}}\left(u \in \frac{1}{2} \mathbb{Z}\right)$ by the sequence of mutations

$$
\begin{aligned}
\cdots \stackrel{\mu_{-}^{\bullet}}{\longrightarrow} & (B, x(0), y(0)) \stackrel{\mu_{+}^{\bullet} \mu_{+}^{\circ}}{\longrightarrow}\left(-B, x\left(\frac{1}{2}\right), y\left(\frac{1}{2}\right)\right) \\
& \stackrel{\mu_{-}^{\bullet}}{\longrightarrow}(\boldsymbol{r}(B), x(1), y(1)) \stackrel{\mu_{+}^{\bullet} \mu_{-}^{\circ}}{\longrightarrow}\left(-\boldsymbol{r}(B), x\left(\frac{3}{2}\right), y\left(\frac{3}{2}\right)\right) \stackrel{\mu_{-}^{\bullet}}{\longrightarrow} \cdots,
\end{aligned}
$$

where $\boldsymbol{r}(B)=B^{\prime}$ is defined by $B_{\mathbf{i j}}^{\prime}=B_{\boldsymbol{r}(\mathbf{i}) \boldsymbol{r}(\mathbf{j})}$.

For a pair $(\mathbf{i}, u) \in \mathbf{I} \times \frac{1}{2} \mathbb{Z}$, we define the parity conditions $\mathbf{p}_{+}$and $\mathbf{p}_{-}$by

$$
\mathbf{p}_{+}:\left\{\begin{array}{ll}
\mathbf{i} \in \mathbf{I}_{+}^{\circ} \sqcup \mathbf{I}_{+}^{\bullet}, & u \equiv 0, \\
\mathbf{i} \in \mathbf{I}_{-}^{\bullet}, & u \equiv \frac{1}{2}, \frac{3}{2}, \\
\mathbf{i} \in \mathbf{I}_{-}^{\circ} \sqcup \mathbf{I}_{+}^{\bullet}, & u \equiv 1,
\end{array} \quad \mathbf{p}_{-}: \begin{cases}\mathbf{i} \in \mathbf{I}_{+}^{\circ} \sqcup \mathbf{I}_{+}^{\bullet}, & u \equiv \frac{1}{2}, \\
\mathbf{i} \in \mathbf{I}_{-}^{\bullet}, & u \equiv 0,1, \\
\mathbf{i} \in \mathbf{I}_{-}^{\circ} \sqcup \mathbf{I}_{+}^{\bullet}, & u \equiv \frac{3}{2},\end{cases}\right.
$$

where $\equiv$ is equivalence modulo $2 \mathbb{Z}$. We have

$$
(\mathbf{i}, u): \mathbf{p}_{+} \Leftrightarrow\left(\mathbf{i}, u+\frac{1}{2}\right): \mathbf{p}_{-} .
$$

Each $(\mathbf{i}, u): \mathbf{p}_{+}$is a mutation point of (3.11) in the forward direction of $u$, and each $(\mathbf{i}, u): \mathbf{p}_{-}$is one in the backward direction of $u$. Notice that there are also 


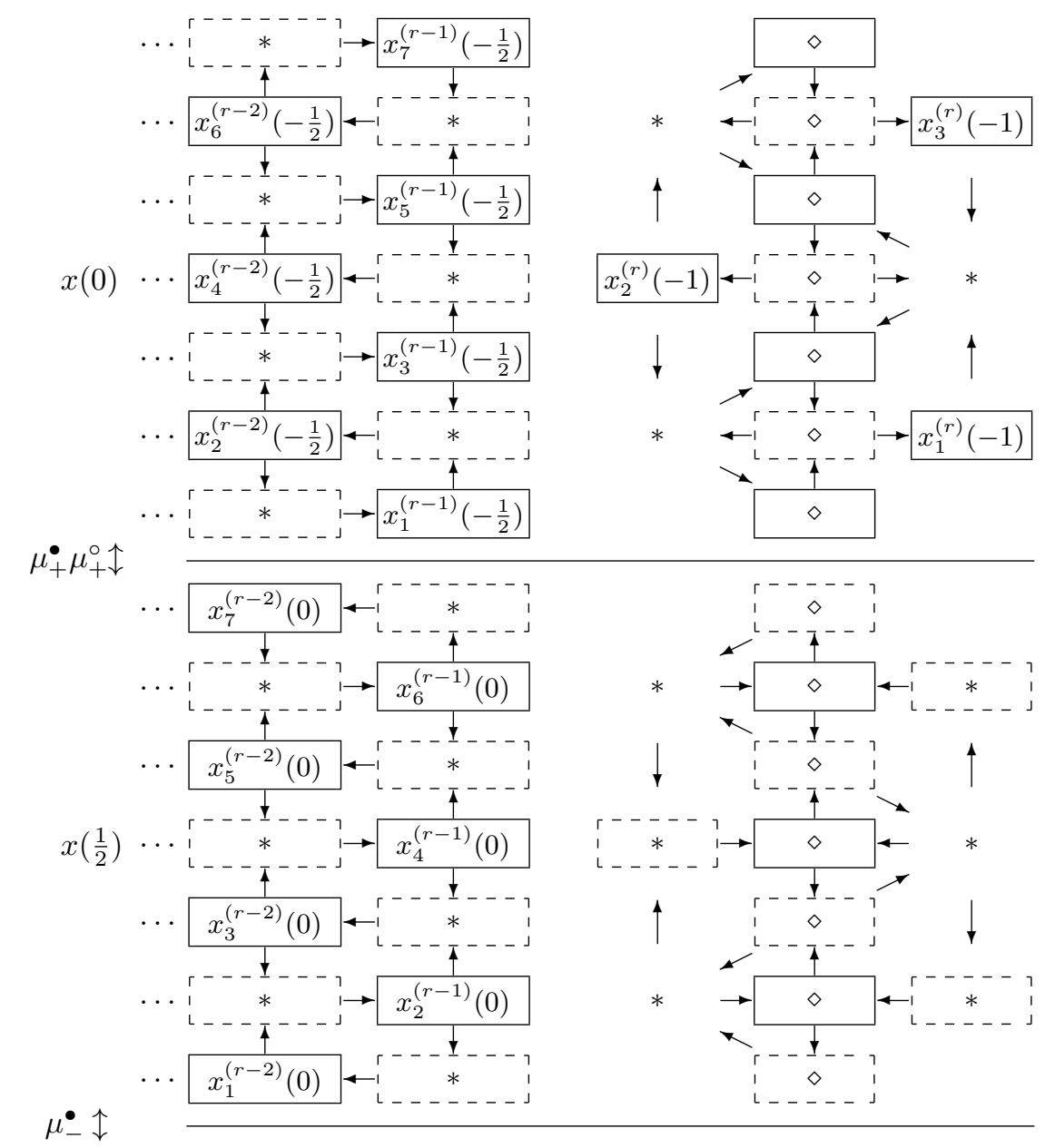

Figure 2. (Continued in Figure 3.) Labeling of cluster variables $x_{\mathbf{i}}(u)$ by $\mathcal{I}_{\ell+}$ for $C_{r}$, $\ell=4$. The variables framed by solid/dashed lines satisfy the condition $\mathbf{p}_{+} / \mathbf{p}_{-}$, respectively. The middle column in the right quiver (marked by $\diamond$ ) is identified with the rightmost column in the left quiver.

some (i, $u)$ which satisfy neither $\mathbf{p}_{+}$nor $\mathbf{p}_{-}$, and are not mutation points of (3.11); explicitly, they are $(\mathbf{i}, u)$ with $\mathbf{i} \in \mathbf{I}_{+}^{\circ}, u \equiv 1, \frac{3}{2} \bmod 2 \mathbb{Z}$, or with $\mathbf{i} \in \mathbf{I}_{-}^{\circ}, u \equiv 0, \frac{1}{2}$ $\bmod 2 \mathbb{Z}$.

There is a correspondence between the parity condition $\mathbf{p}_{ \pm}$here and $\mathbf{P}_{ \pm}, \mathbf{P}_{ \pm}^{\prime}$ in (3.1) and (3.4). 


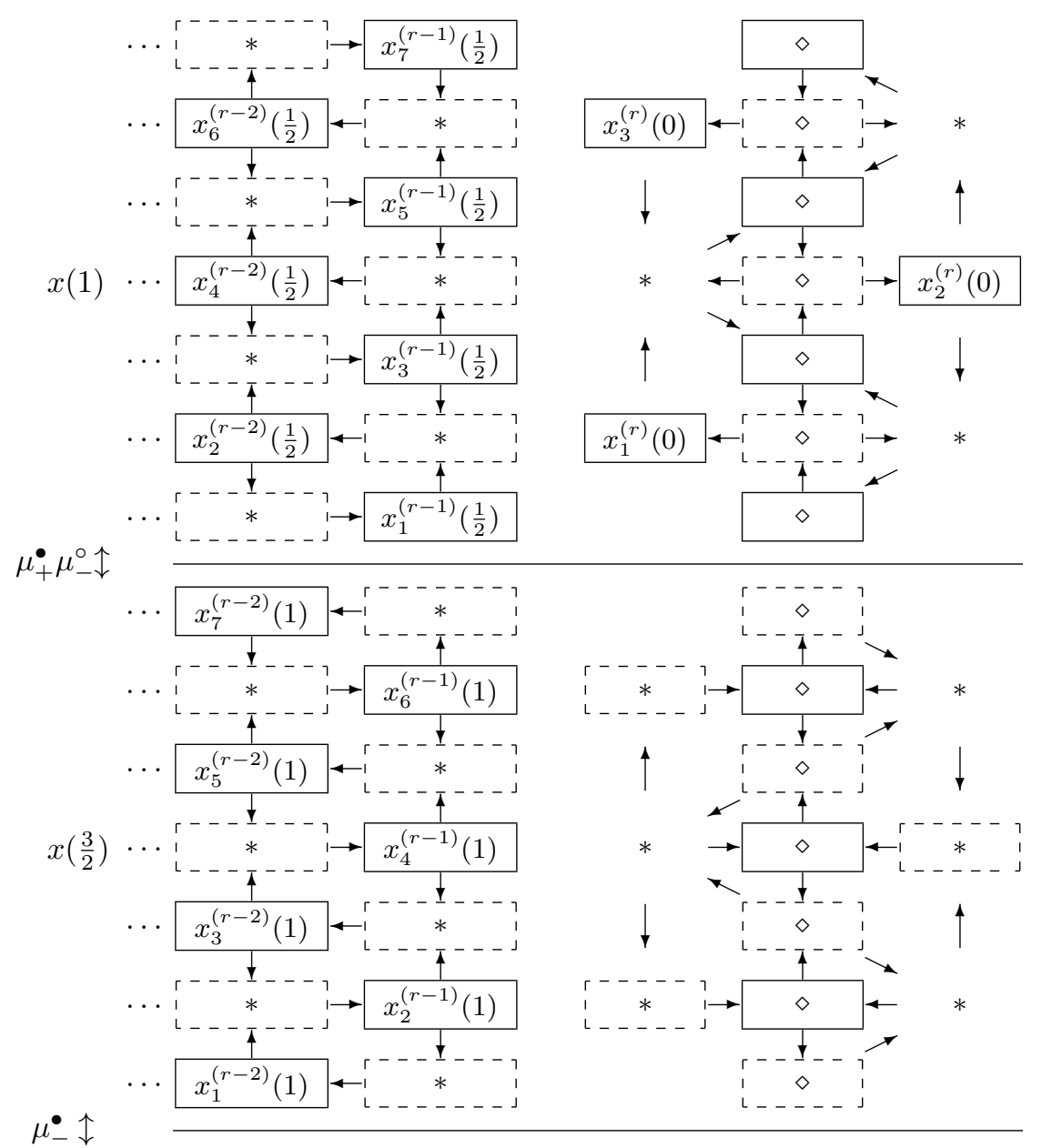

Figure 3. Continuation of Figure 2.

Lemma 3.3. Below $\equiv$ means equivalence modulo $2 \mathbb{Z}$.

(i) The map $g: \mathcal{I}_{\ell+} \rightarrow\left\{(\mathbf{i}, u): \mathbf{p}_{+}\right\}$given by

$$
\left(a, m, u-\frac{1}{t_{a}}\right) \mapsto \begin{cases}((a, m), u), & a \neq r, \\ ((r+1, m), u), & a=r ; m+u \equiv 0, \\ ((r, m), u), & a=r ; m+u \equiv 1,\end{cases}
$$

is a bijection. 
(ii) The map $g^{\prime}: \mathcal{I}_{\ell+}^{\prime} \rightarrow\left\{(\mathbf{i}, u): \mathbf{p}_{+}\right.$or $\left.\mathbf{p}_{-}\right\}$given by

$$
(a, m, u) \mapsto \begin{cases}((a, m), u), & a \neq r, \\ ((r+1, m), u), & a=r ; m+u \equiv 0, \\ ((r, m), u), & a=r ; m+u \equiv 1,\end{cases}
$$

is a bijection.

We introduce alternative labels $x_{\mathbf{i}}(u)=x_{m}^{(a)}\left(u-1 / t_{a}\right)\left(\left(a, m, u-1 / t_{a}\right) \in \mathcal{I}_{\ell+}\right)$ for $(\mathbf{i}, u)=g\left(\left(a, m, u-1 / t_{a}\right)\right)$ and $y_{\mathbf{i}}(u)=y_{m}^{(a)}(u)\left((a, m, u) \in \mathcal{I}_{\ell+}^{\prime}\right)$ for $(\mathbf{i}, u)=$ $g^{\prime}((a, m, u))$, respectively. See Figures $2-3$.

\section{§3.4. T-system and cluster algebra}

The result in this subsection is completely parallel to the $B_{r}$ case [IIKKN].

Let $\mathcal{A}(B, x)$ be the cluster algebra with trivial coefficients, where $(B, x)$ is the initial seed [FZ2]. Let $\mathbf{1}=\{1\}$ be the trivial semifield and $\pi_{\mathbf{1}}: \mathbb{Q}_{\mathrm{sf}}(y) \rightarrow \mathbf{1}$, $y_{\mathbf{i}} \mapsto 1$, be the projection. Let $\left[x_{\mathbf{i}}(u)\right]_{\mathbf{1}}$ denote the image of $x_{\mathbf{i}}(u)$ under the algebra homomorphism $\mathcal{A}(B, x, y) \rightarrow \mathcal{A}(B, x)$ induced by $\pi_{\mathbf{1}}$. It is called the trivial evaluation.

Recall that $G(b, k, v ; a, m, u)$ is defined in (2.10).

Lemma 3.4. The family $\left\{x_{m}^{(a)}(u) \mid(a, m, u) \in \mathcal{I}_{\ell+}\right\}$ satisfies a system of relations

$$
\begin{aligned}
x_{m}^{(a)}\left(u-\frac{1}{t_{a}}\right) x_{m}^{(a)}\left(u+\frac{1}{t_{a}}\right)= & \frac{y_{m}^{(a)}(u)}{1+y_{m}^{(a)}(u)} \prod_{(b, k, v) \in \mathcal{I}_{\ell+}} x_{k}^{(b)}(v)^{G(b, k, v ; a, m, u)} \\
& +\frac{1}{1+y_{m}^{(a)}(u)} x_{m-1}^{(a)}(u) x_{m+1}^{(a)}(u),
\end{aligned}
$$

where $(a, m, u) \in \mathcal{I}_{\ell+}^{\prime}$. In particular, the family $\left\{\left[x_{m}^{(a)}(u)\right]_{\mathbf{1}} \mid(a, m, u) \in \mathcal{I}_{\ell+}\right\}$ satisfies the T-system $\mathbb{T}_{\ell}\left(C_{r}\right)$ in $\mathcal{A}(B, x)$ after replacing $T_{m}^{(a)}(u)$ with $\left[x_{m}^{(a)}(u)\right]_{\mathbf{1}}$.

Definition 3.5. The T-subalgebra $\mathcal{A}_{T}(B, x)$ of $\mathcal{A}(B, x, y)$ associated with the sequence (3.11) is the subalgebra of $\mathcal{A}(B, x)$ generated by $\left[x_{\mathbf{i}}(u)\right]_{\mathbf{1}}\left((\mathbf{i}, u) \in \mathbf{I} \times \frac{1}{2} \mathbb{Z}\right)$.

Theorem 3.6. The ring $\mathcal{T}_{\ell}^{\circ}\left(C_{r}\right)_{+}$is isomorphic to $\mathcal{A}_{T}(B, x)$ via the correspondence $T_{m}^{(a)}(u) \mapsto\left[x_{m}^{(a)}(u)\right]_{\mathbf{1}}$.

\section{$\S 3.5$. Y-system and cluster algebra}

The result in this subsection is completely parallel to the $B_{r}$ case [IIKKN]. 
Lemma 3.7. The family $\left\{y_{m}^{(a)}(u) \mid(a, m, u) \in \mathcal{I}_{\ell+}^{\prime}\right\}$ satisfies the $Y$-system $\mathbb{Y}_{\ell}\left(C_{r}\right)$ after replacing $Y_{m}^{(a)}(u)$ with $y_{m}^{(a)}(u)$.

Definition 3.8. The $Y$-subgroup $\mathcal{G}_{Y}(B, y)$ of $\mathcal{G}(B, y)$ associated with the sequence (3.11) is the subgroup of $\mathcal{G}(B, y)$ generated by $y_{\mathbf{i}}(u)\left((\mathbf{i}, u) \in \mathbf{I} \times \frac{1}{2} \mathbb{Z}\right)$ and $1+y_{\mathbf{i}}(u)$ $\left((\mathbf{i}, u): \mathbf{p}_{+}\right.$or $\left.\mathbf{p}_{-}\right)$.

Theorem 3.9. The group $y_{\ell}^{\circ}\left(C_{r}\right)_{+}$is isomorphic to $\mathcal{G}_{Y}(B, y)$ via the correspondence $Y_{m}^{(a)}(u) \mapsto y_{m}^{(a)}(u)$ and $1+Y_{m}^{(a)}(u) \mapsto 1+y_{m}^{(a)}(u)$.

\section{§3.6. Tropical Y-system at level 2}

The tropical semifield $\operatorname{Trop}(y)$ is an abelian multiplicative group freely generated by the elements $y_{\mathbf{i}}(\mathbf{i} \in \mathbf{I})$ with addition

$$
\prod_{\mathbf{i} \in \mathbf{I}} y_{\mathbf{i}}^{a_{\mathbf{i}}} \oplus \prod_{\mathbf{i} \in \mathbf{I}} y_{\mathbf{i}}^{b_{\mathbf{i}}}=\prod_{\mathbf{i} \in \mathbf{I}} y_{\mathbf{i}}^{\min \left(a_{\mathbf{i}}, b_{\mathbf{i}}\right)} .
$$

Let $\pi_{\mathbf{T}}: \mathbb{Q}_{\mathrm{sf}}(y) \rightarrow \operatorname{Trop}(y), y_{\mathbf{i}} \mapsto y_{\mathbf{i}}$, be the projection. Let $\left[y_{\mathbf{i}}(u)\right]_{\mathbf{T}}$ and $\left[\mathcal{G}_{Y}(B, y)\right]_{\mathbf{T}}$ denote the images of $y_{\mathbf{i}}(u)$ and $\mathcal{G}_{Y}(B, y)$ under the multiplicative group homomorphism induced by $\pi_{\mathbf{T}}$, respectively. They are called the tropical evaluations, and the resulting relations in the group $\left[\mathcal{G}_{Y}(B, y)\right]_{\mathbf{T}}$ form the tropical $Y$-system.

We say a (Laurent) monomial $m=\prod_{\mathbf{i} \in \mathbf{I}} y_{\mathbf{i}}^{k_{\mathbf{i}}}$ is positive (resp. negative) if $m \neq 1$ and $k_{\mathbf{i}} \geq 0$ (resp. $k_{\mathbf{i}} \leq 0$ ) for any $\mathbf{i}$.

The following properties of the tropical Y-system at level 2 will be the key in the entire method.

Proposition 3.10. For $\left[\mathcal{G}_{Y}(B, y)\right]_{\mathbf{T}}$ with $B=B_{2}\left(C_{r}\right)$, the following facts hold:

(i) Let $0 \leq u<2$. For any $(\mathbf{i}, u): \mathbf{p}_{+}$, the monomial $\left[y_{\mathbf{i}}(u)\right]_{\mathbf{T}}$ is positive.

(ii) Let $-h^{\vee} \leq u<0$.

(a) Let $\mathbf{i}=(i, 2)(i \leq r-1),(r, 1)$, or $(r+1,1)$. For any $(\mathbf{i}, u): \mathbf{p}_{+}$, the monomial $\left[y_{\mathbf{i}}(u)\right]_{\mathbf{T}}$ is negative.

(b) Let $\mathbf{i}=(i, 1),(i, 3)(i \leq r-1)$. For any $(\mathbf{i}, u): \mathbf{p}_{+}$, the monomial $\left[y_{\mathbf{i}}(u)\right]_{\mathbf{T}}$ is positive for $u=-\frac{1}{2} h^{\vee},-\frac{1}{2} h^{\vee}-\frac{1}{2}$ and negative otherwise.

(iii) $y_{i i^{\prime}}(2)$ equals $y_{i, 4-i^{\prime}}^{-1}$ if $i \leq r-1$, and $y_{i i^{\prime}}^{-1}$ if $i=r, r+1$.

(iv) For even $r, y_{i i^{\prime}}\left(-h^{\vee}\right)$ equals $y_{i i^{\prime}}^{-1}$ if $i \leq r-1$, and $y_{2 r+1-i, i^{\prime}}^{-1}$ if $i=r, r+1$. For odd $r, y_{i i^{\prime}}\left(-h^{\vee}\right)=y_{i i^{\prime}}^{-1}$.

One can directly verify (i) and (iii) in the same way as in the $B_{r}$ case [IIKKN, Proposition 3.2]. In the rest of this subsection we give the outline of the proof 
of (ii) and (iv). Note that (ii) and (iv) can be proved independently for each variable $y_{\mathbf{i}}$. (To be precise, we also need to ensure that no monomial is 1 . However, this can be easily guaranteed, so that we do not describe the details here.) Below we separate the variables into two parts. Here is a brief summary of the results.

(1) The $D$ part. The powers of $\left[y_{\mathbf{i}}(u)\right]_{\mathbf{T}}$ in the variables $y_{i, 2}(i \leq r-1)$ and $y_{r, 1}, y_{r+1,1}$ are described by the root system of type $D_{r+1}$ with a Coxeter-like transformation. It turns out that they are further described by (a subset of) the root system of type $A_{2 r+1}$ with the Coxeter transformation.

(2) The $A$ part. The powers of $\left[y_{\mathbf{i}}(u)\right]_{\mathbf{T}}$ in the variables $y_{i, 1}$ and $y_{i, 3}(i \leq$ $r-1)$ are mainly described by the root system of type $A_{r-1}$ with the Coxeter transformation.

3.6.1. D part. Let us consider the $D$ part first. Let $D_{r+1}$ be the Dynkin diagram of type $D$ with index set $J=\{1, \ldots, r+1\}$. We assign a sign $+/-$ to vertices of $D_{r+1}$ (no sign for $r$ and $r+1$ ) as inherited from $Q_{2}\left(C_{r}\right)$.

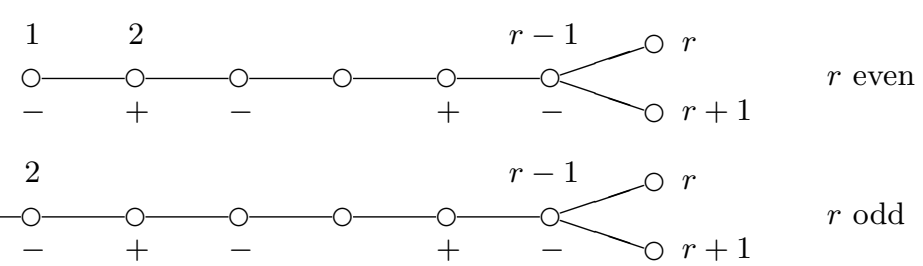

Let $\Pi=\left\{\alpha_{1}, \ldots, \alpha_{r+1}\right\},-\Pi, \Phi_{+}$be the sets of simple roots, of negative simple roots, and of positive roots, respectively, of type $D_{r+1}$. Following [FZ1], we introduce the piecewise-linear analogue $\sigma_{i}$ of the simple reflection $s_{i}$, acting on the set $\Phi_{\geq-1}=\Phi_{+} \sqcup(-\Pi)$ of almost positive roots by

$$
\begin{gathered}
\sigma_{i}(\alpha)=s_{i}(\alpha), \quad \alpha \in \Phi_{+}, \\
\sigma_{i}\left(-\alpha_{j}\right)= \begin{cases}\alpha_{j}, & j=i, \\
-\alpha_{j}, & \text { otherwise }\end{cases}
\end{gathered}
$$

Let

$$
\sigma_{+}=\prod_{i \in J_{+}} \sigma_{i}, \quad \sigma_{-}=\prod_{i \in J_{-}} \sigma_{i}
$$

where $J_{ \pm}$is the set of vertices of $D_{r+1}$ with the respective sign. We define

$$
\sigma=\sigma_{-} \sigma_{+} \sigma_{r+1} \sigma_{-} \sigma_{+} \sigma_{r} .
$$


Lemma 3.11. The following facts hold:

(I) Let $r$ be even.

(i) For $i \leq r-1, \sigma^{k}\left(-\alpha_{i}\right) \in \Phi_{+}(1 \leq k \leq r / 2), \sigma^{r / 2+1}\left(-\alpha_{i}\right)=-\alpha_{i}$.

(ii) For $i \leq r-1, \sigma^{k}\left(\alpha_{i}\right) \in \Phi_{+}(0 \leq k \leq r / 2), \sigma^{r / 2+1}\left(\alpha_{i}\right)=\alpha_{i}$.

(iii) $\sigma^{k}\left(-\alpha_{r}\right) \in \Phi_{+}(1 \leq k \leq r / 2), \sigma^{r / 2+1}\left(-\alpha_{r}\right)=-\alpha_{r+1}$.

(iv) $\sigma^{k}\left(-\alpha_{r+1}\right) \in \Phi_{+}(1 \leq k \leq r / 2+1), \sigma^{r / 2+2}\left(-\alpha_{r+1}\right)=-\alpha_{r}$.

(v) The elements of $\Phi_{+}$appearing in (i)-(iv) exhaust the set $\Phi_{+}$, thereby providing the orbit decomposition of $\Phi_{+}$under $\sigma$.

(II) Let $r$ be odd.

(i) For $i \in J_{+}, \sigma^{k}\left(-\alpha_{i}\right) \in \Phi_{+}(1 \leq k \leq r+1), \sigma^{r+2}\left(-\alpha_{i}\right)=-\alpha_{i}$, $\sigma^{(r+1) / 2}\left(-\alpha_{i}\right)=\alpha_{i}$.

(ii) For $i \in J_{-}, \sigma^{k}\left(-\alpha_{i}\right) \in \Phi_{+}(1 \leq k \leq r+1), \sigma^{r+2}\left(-\alpha_{i}\right)=-\alpha_{i}$, $\sigma^{(r+3) / 2}\left(-\alpha_{i}\right)=\alpha_{i}$.

(iii) $\sigma^{k}\left(-\alpha_{r}\right) \in \Phi_{+}(1 \leq k \leq(r+1) / 2), \sigma^{(r+3) / 2}\left(-\alpha_{r}\right)=-\alpha_{r}$.

(iv) $\sigma^{k}\left(-\alpha_{r+1}\right) \in \Phi_{+}(1 \leq k \leq(r+1) / 2), \sigma^{(r+3) / 2}\left(-\alpha_{r+1}\right)=-\alpha_{r+1}$.

(v) The elements of $\Phi_{+}$appearing in (i)-(iv) exhaust the set $\Phi_{+}$, thereby providing the orbit decomposition of $\Phi_{+}$under $\sigma$.

Proof. The statements are verified by explicitly calculating $\sigma^{k}\left(-\alpha_{i}\right)$ and $\sigma^{k}\left(\alpha_{i}\right)$. The examples for $r=10$ (for even $r$ ) and 9 (for odd $r$ ) are given in Tables 1 and 2, respectively, where we use the notations

$$
\begin{aligned}
{[i, j] } & =\alpha_{i}+\cdots+\alpha_{j} \quad(1 \leq i<j \leq r), \quad[i]=\alpha_{i} \quad(1 \leq i \leq r), \\
\{i, j\} & =\left(\alpha_{i}+\cdots+\alpha_{r-1}\right)+\left(\alpha_{j}+\cdots+\alpha_{r+1}\right) \quad(1 \leq i<j \leq r+1, i \leq r-1),
\end{aligned}
$$

and $\{r+1\}=\alpha_{r+1}$. In fact, it is not difficult to read off the general rule from these examples.

The orbits $\sigma\left(-\alpha_{i}\right)$ and $\sigma\left(\alpha_{i}\right)$ are further described by (a subset of) the root system of type $A_{2 r+1}$. Let $\Pi^{\prime}=\left\{\alpha_{1}^{\prime}, \ldots, \alpha_{2 r+1}^{\prime}\right\}$ and $\Phi_{+}^{\prime}$ be the sets of simple roots and of positive roots of type $A_{2 r+1}$, respectively, with standard index set $J^{\prime}=\{1, \ldots, 2 r+1\}$. Define $J_{+}^{\prime}=\left\{i \in J^{\prime} \mid i-r\right.$ is even $\}$ and $J_{-}^{\prime}=\left\{i \in J^{\prime} \mid\right.$ $i-r$ is odd $\}$. We introduce the notations $[i, j]^{\prime}=\alpha_{i}^{\prime}+\cdots+\alpha_{j}^{\prime}(1 \leq i<j \leq 2 r+1)$ and $[i]^{\prime}=\alpha_{i}^{\prime}$, parallel to (3.21). Let $O_{i}^{\prime}=\left\{\left(\sigma^{\prime}\right)^{k}\left(-\alpha_{i}^{\prime}\right) \mid 1 \leq k \leq r+1\right\}$ be the orbit of $-\alpha_{i}^{\prime}$ in $\Phi_{+}^{\prime}$ under $\sigma^{\prime}=\sigma_{-}^{\prime} \sigma_{+}^{\prime}, \sigma_{ \pm}^{\prime}=\prod_{i \in J_{+}^{\prime}} \sigma_{i}^{\prime}$, where $\sigma_{i}^{\prime}$ is the piecewise-linear analogue of the simple reflection $s_{i}^{\prime}$ acting as in (3.18). 


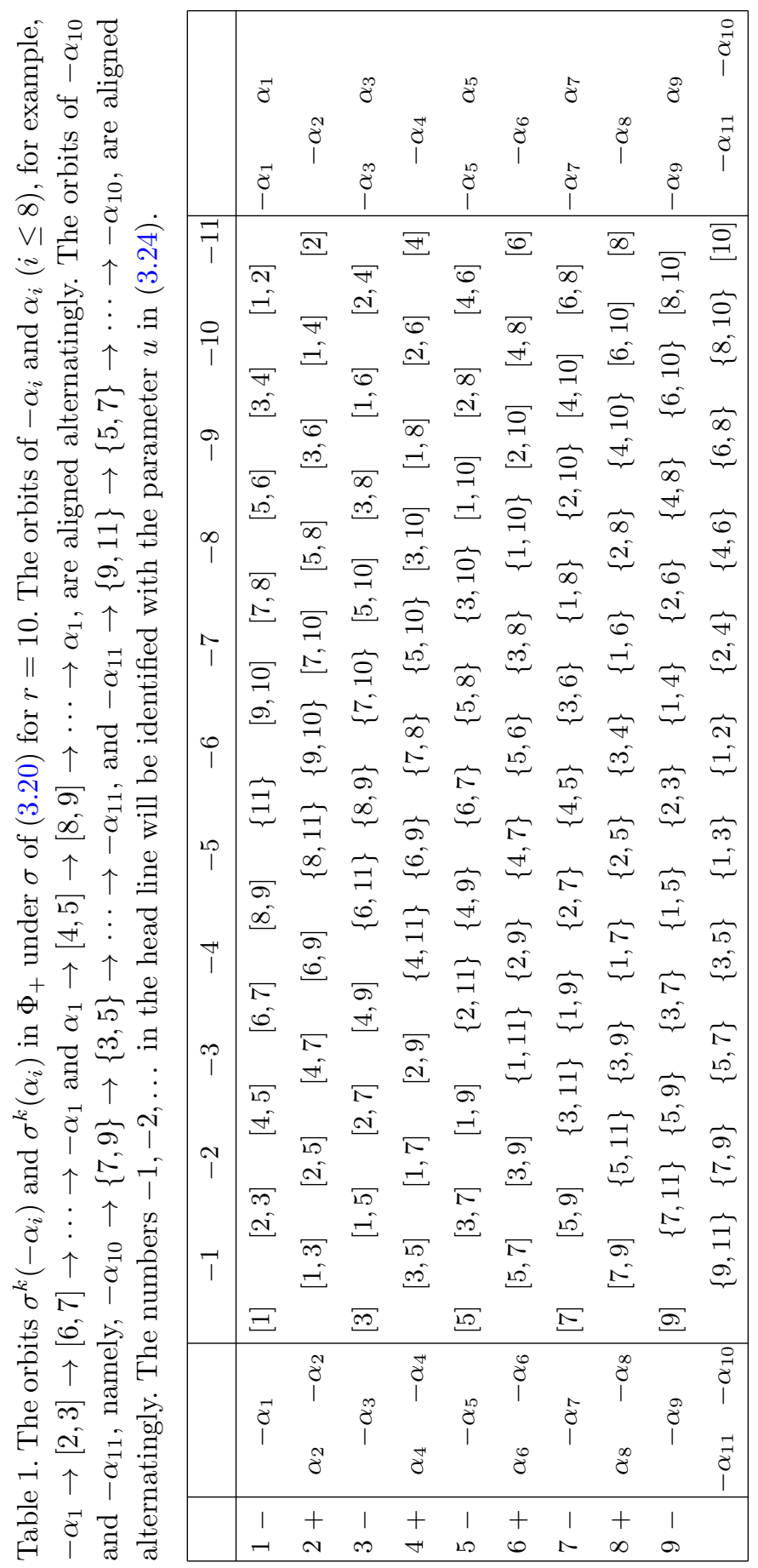


Periodicities of T-systems and Y-systems II: Types $C_{r}, F_{4}$, And $G_{2}$

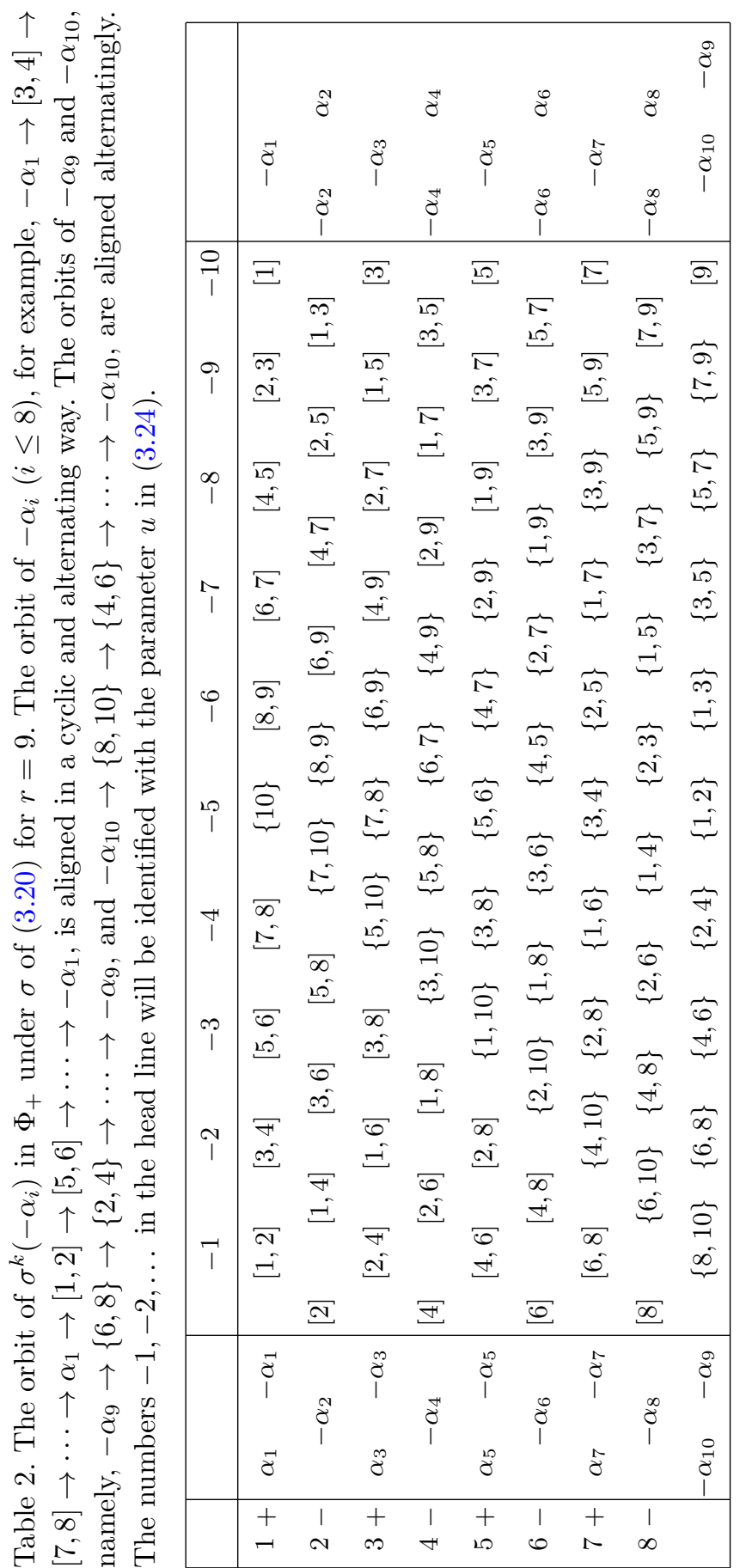


Lemma 3.12. Let

$$
\rho: \Phi_{+} \rightarrow \bigsqcup_{i=1}^{r} O_{i}^{\prime}
$$

be the map defined by

$$
\begin{aligned}
{[i, j] } & \mapsto \begin{cases}{[i, j]^{\prime},} & j-r \text { odd }, \\
{[2 r+2-j, 2 r+2-i]^{\prime},} & j-r \text { even, }\end{cases} \\
\{i, j\} & \mapsto \begin{cases}{[i, 2 r+2-j]^{\prime},} & j-r \text { odd, } \\
{[j, 2 r+2-i]^{\prime},} & j-r \text { even, }\end{cases} \\
\{r+1\} & \mapsto[r, r+1]^{\prime},
\end{aligned}
$$

where $[i]=[i, i]$. Then $\rho$ is a bijection. Furthermore, under the bijection $\rho$, the action of $\sigma$ is translated into the one of the square of the Coxeter element $s^{\prime}=s_{-}^{\prime} s_{+}^{\prime}$ of type $A_{2 r+1}$ acting on $\Phi_{+}^{\prime}$, where $s_{ \pm}^{\prime}=\prod_{i \in J_{ \pm}^{\prime}} s_{i}^{\prime}$.

For $-h^{\vee} \leq u<0$, define

$$
\alpha_{i}(u)= \begin{cases}\sigma^{-u / 2}\left(-\alpha_{i}\right), & i \in J_{+}, u \equiv 0, \\ \sigma^{-(u-1) / 2}\left(\alpha_{i}\right), & i \in J_{+}, u \equiv-1, \\ \sigma^{-(2 u-1) / 4}\left(-\alpha_{i}\right), & i \in J_{-}, u \equiv-\frac{3}{2}, \\ \sigma^{-(2 u+1) / 4}\left(\alpha_{i}\right), & i \in J_{-}, u \equiv-\frac{1}{2}, \\ \sigma^{-u / 2}\left(-\alpha_{r}\right), & i=r, u \equiv 0, \\ \sigma^{-(u-1) / 2}\left(-\alpha_{r+1}\right), & i=r+1, u \equiv-1,\end{cases}
$$

where $\equiv$ is equivalence modulo $2 \mathbb{Z}$. Note that they correspond to the positive roots in Tables 1 and 2 with $u$ being the parameter in the head lines. By Lemma 3.11 they are all the positive roots of $D_{r+1}$.

Lemma 3.13. The family in (3.24) satisfies the recurrence relations

$$
\begin{aligned}
\alpha_{i}\left(u-\frac{1}{2}\right)+\alpha_{i}\left(u+\frac{1}{2}\right) & =\alpha_{i-1}(u)+\alpha_{i+1}(u) \quad(1 \leq i \leq r-2), \\
\alpha_{r-1}\left(u-\frac{1}{2}\right)+\alpha_{r-1}\left(u+\frac{1}{2}\right) & = \begin{cases}\alpha_{r-2}(u)+\alpha_{r}(u) \quad(u \text { even }), \\
\alpha_{r-2}(u)+\alpha_{r+1}(u) & (u \text { odd }),\end{cases} \\
\alpha_{r}(u-1)+\alpha_{r}(u+1) & =\alpha_{r-1}\left(u-\frac{1}{2}\right)+\alpha_{r-1}\left(u+\frac{1}{2}\right) \quad(u \text { odd }), \\
\alpha_{r+1}(u-1)+\alpha_{r+1}(u+1) & =\alpha_{r-1}\left(u-\frac{1}{2}\right)+\alpha_{r-1}\left(u+\frac{1}{2}\right) \quad(u \text { even }),
\end{aligned}
$$

where $\alpha_{0}(u)=0$. 
Proof. These relations are easily verified by using the explicit expressions of $\alpha_{i}(u)$. See Tables 1 and 2. The first two relations can also be obtained from Lemma 3.12 and [FZ2, Eq. (10.9)].

Let us return to the proof of (ii) of Proposition 3.10 for the $D$ part. For a monomial $m$ in $y=\left(y_{\mathbf{i}}\right)_{\mathbf{i} \in \mathbf{I}}$, let $\pi_{D}(m)$ denote the specialization with $y_{i, 1}=y_{i, 3}=1$ $(i \leq r-1)$. For simplicity, we set $y_{i 2}=y_{i}(i \leq r-1), y_{r 1}=y_{r}, y_{r+1,1}=y_{r+1}$, and also $y_{i 2}(u)=y_{i}(u)(i \leq r-1), y_{r 1}(u)=y_{r}(u), y_{r+1,1}(u)=y_{r}(u)$. We define the vectors $\mathbf{t}_{i}(u)=\left(t_{i}(u)_{k}\right)_{k=1}^{r+1}$ by

$$
\pi_{D}\left(\left[y_{i}(u)\right]_{\mathbf{T}}\right)=\prod_{k=1}^{r+1} y_{k}^{t_{i}(u)_{k}} .
$$

We also identify each vector $\mathbf{t}_{i}(u)$ with $\alpha=\sum_{k=1}^{r+1} t_{i}(u)_{k} \alpha_{k} \in \mathbb{Z} \Pi$.

Proposition 3.14. Let $-h^{\vee} \leq u<0$. Then

$$
\mathbf{t}_{i}(u)=-\alpha_{i}(u)
$$

for $(i, u)$ as in (3.24), and

$$
\pi_{D}\left(\left[y_{i 1}(u)\right]_{\mathbf{T}}\right)=\pi_{D}\left(\left[y_{i 3}(u)\right]_{\mathbf{T}}\right)=1 \quad(i \leq r-1, r+i+2 u \text { even }) .
$$

Note that these formulas determine $\pi_{D}\left(\left[y_{\mathbf{i}}(u)\right]_{\mathbf{T}}\right)$ for any $(\mathbf{i}, u): \mathbf{p}_{+}$.

Proof. We can verify the claim for $-2 \leq u \leq-\frac{1}{2}$ by direct computation. Then, by backward induction on $u$, one can establish the claim, together with the recurrence relations among $\mathbf{t}_{i}(u)$ 's with $(i, u)$ in (3.24),

$$
\begin{aligned}
\mathbf{t}_{i}\left(u-\frac{1}{2}\right)+\mathbf{t}_{i}\left(u+\frac{1}{2}\right) & =\mathbf{t}_{i-1}(u)+\mathbf{t}_{i+1}(u) \quad(1 \leq i \leq r-2), \\
\mathbf{t}_{r-1}\left(u-\frac{1}{2}\right)+\mathbf{t}_{r-1}\left(u+\frac{1}{2}\right) & = \begin{cases}\mathbf{t}_{r-2}(u)+\mathbf{t}_{r}(u) & (u \text { even }), \\
\mathbf{t}_{r-2}(u)+\mathbf{t}_{r+1}(u) & (u \text { odd }),\end{cases} \\
\mathbf{t}_{r}(u-1)+\mathbf{t}_{r}(u+1) & =\mathbf{t}_{r-1}\left(u-\frac{1}{2}\right)+\mathbf{t}_{r-1}\left(u+\frac{1}{2}\right) \quad(u \text { odd }), \\
\mathbf{t}_{r+1}(u-1)+\mathbf{t}_{r+1}(u+1) & =\mathbf{t}_{r-1}\left(u-\frac{1}{2}\right)+\mathbf{t}_{r-1}\left(u+\frac{1}{2}\right) \quad(u \text { even }) .
\end{aligned}
$$

Note that (3.29) coincides with (3.25) under (3.27). To derive (3.29), one uses the mutations as in [IIKKN, Figure 6] (or the tropical version of the Y-system $\mathbb{Y}_{2}\left(C_{r}\right)$ directly) and the positivity/negativity of $\pi_{D}\left(\left[y_{\mathbf{i}}(u)\right]_{\mathbf{T}}\right)$ resulting from (3.27) and (3.28) by induction hypothesis.

Now (ii) and (iv) of Proposition 3.10 for the $D$ part follow from Proposition 3.14.

3.6.2. A part. The $A$ part can be studied in a similar way to the $D$ part. Therfore, we only present the result. 
First we note that the quiver $Q_{2}\left(C_{r}\right)$ is symmetric under the exchange $y_{i, 1} \leftrightarrow$ $y_{i, 3}(i \leq r-1)$. Thus, one can concentrate on the powers of $\left[y_{\mathbf{i}}(u)\right]_{\mathbf{T}}$ in the variables $y_{i, 1}(i \leq r-1)$.

Let $A_{r-1}$ be the Dynkin diagram of type $A$ with index set $J=\{1, \ldots, r-1\}$. We assign a sign $+/-$ to vertices (except for $r$ ) of $A_{r-1}$ as inherited from $Q_{2}\left(C_{r}\right)$ :

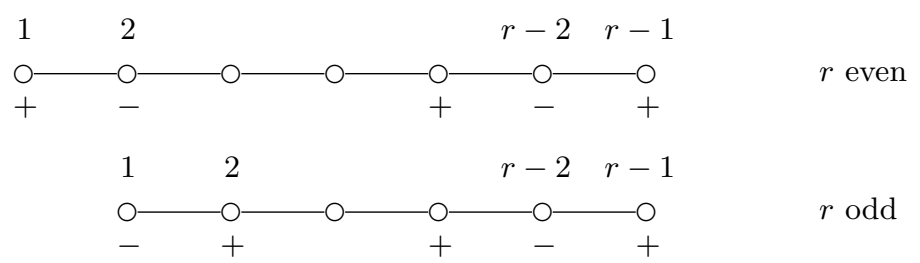

Let $\Pi=\left\{\alpha_{1}, \ldots, \alpha_{r-1}\right\},-\Pi, \Phi_{+}$be the sets of simple roots, of negative simple roots, and of positive roots, respectively, of type $A_{r-1}$. Again, we introduce the piecewise-linear analogue $\sigma_{i}$ of the simple reflection $s_{i}$, acting on $\Phi_{\geq-1}=$ $\Phi_{+} \sqcup(-\Pi)$ as in (3.18). Let

$$
\sigma_{+}=\prod_{i \in J_{+}} \sigma_{i}, \quad \sigma_{-}=\prod_{i \in J_{-}} \sigma_{i}
$$

where $J_{ \pm}$is the set of vertices of $A_{r-1}$ with the respective sign. We define

$$
\sigma=\sigma_{-} \sigma_{+}
$$

For a monomial $m$ in $y=\left(y_{\mathbf{i}}\right)_{\mathbf{i} \in \mathbf{I}}$, let $\pi_{A}(m)$ denote the specialization with $y_{i, 2}=y_{i, 3}=1(i \leq r-1)$ and $y_{r 1}=y_{r+1,1}=1$. We set $y_{i 1}=y_{i}(i \leq r-1)$.

We define the vectors $\mathbf{t}_{\mathbf{i}}(u)=\left(t_{\mathbf{i}}(u)_{k}\right)_{k=1}^{r-1}$ by

$$
\pi_{A}\left(\left[y_{\mathbf{i}}(u)\right]_{\mathbf{T}}\right)=\prod_{k=1}^{r-1} y_{k}^{t_{\mathbf{i}}(u)_{k}} .
$$

We also identify each vector $\mathbf{t}_{\mathbf{i}}(u)$ with $\alpha=\sum_{k=1}^{r-1} t_{\mathbf{i}}(u)_{k} \alpha_{k} \in \mathbb{Z} \Pi$.

With these notations, the result for the $A$ part is summarized as follows.

Proposition 3.15. Let $-h^{\vee} \leq u<0$. For $(\mathbf{i}, u): \mathbf{p}_{+}, \mathbf{t}_{\mathbf{i}}(u)$ is given by, for $i \leq r-1$,

$$
\begin{aligned}
& \mathbf{t}_{i 1}(u)= \begin{cases}-\sigma^{-u}\left(-\alpha_{i}\right), & i \in J_{+}, \\
-\sigma^{-(2 u-1) / 2}\left(-\alpha_{i}\right), & i \in J_{-},\end{cases} \\
& \mathbf{t}_{i 2}(u)= \begin{cases}-[2 r+2-i+2 u, r-1], & -\frac{1}{2} h^{\vee} \leq u<0, \\
-[-1-i-2 u, r-1], & -h^{\vee} \leq u<-\frac{1}{2} h^{\vee},\end{cases} \\
& \mathbf{t}_{i 3}(u)=0,
\end{aligned}
$$


and

$$
\begin{gathered}
\mathbf{t}_{r 1}(u)= \begin{cases}-[r+2+2 u, r-1], & -\frac{1}{2} h^{\vee} \leq u<0, \\
-[-1-r-2 u, r-1], & -h^{\vee} \leq u<-\frac{1}{2} h^{\vee},\end{cases} \\
\mathbf{t}_{r+1,1}(u)= \begin{cases}-[r+2+2 u, r-1], & -\frac{1}{2} h^{\vee} \leq u<0, \\
-[-1-r-2 u, r-1], & -h^{\vee} \leq u<-\frac{1}{2} h^{\vee},\end{cases}
\end{gathered}
$$

where $[i, j]$ equals $\alpha_{i}+\cdots+\alpha_{j}$ if $i \leq j$ and 0 if $i>j$.

Note that $\mathbf{t}_{i 1}\left(-h^{\vee} / 2\right)=\alpha_{r-i}\left(i \in J_{-}\right.$for $r$ even and $i \in J_{+}$for $r$ odd $)$ and $\mathbf{t}_{i 1}\left(-h^{\vee} / 2-1 / 2\right)=\alpha_{r-i}\left(i \in J_{+}\right.$for $r$ even and $i \in J_{-}$for $r$ odd $)$, and that they are the only positive monomials in (3.33) and (3.34). Now (ii) and (iv) of Proposition 3.10 for the $A$ part follow from Proposition 3.15.

This completes the proof of Proposition 3.10.

\section{§3.7. Tropical Y-systems at higher levels}

By the same method as for the $B_{r}$ case [IIKKN, Proposition 4.1], one can establish the 'factorization property' of the tropical Y-system at higher levels. As a result, we obtain a generalization of Proposition 3.10.

Proposition 3.16. For $\left[\mathcal{G}_{Y}(B, y)\right]_{\mathbf{T}}$ with $B=B_{\ell}\left(C_{r}\right)$, the following facts hold:

(i) Let $0 \leq u<\ell$. For any $(\mathbf{i}, u)$ : $\mathbf{p}_{+}$, the monomial $\left[y_{\mathbf{i}}(u)\right]_{\mathbf{T}}$ is positive.

(ii) Let $-h^{\vee} \leq u<0$.

(a) Let $\mathbf{i} \in \mathbf{I}^{\circ}$ or $\mathbf{i}=\left(i, i^{\prime}\right)\left(i \leq r-1, i^{\prime} \in 2 \mathbb{N}\right)$. For any $(\mathbf{i}, u): \mathbf{p}_{+}$, the monomial $\left[y_{\mathbf{i}}(u)\right]_{\mathbf{T}}$ is negative.

(b) Let $\mathbf{i}=\left(i, i^{\prime}\right)\left(i \leq r-1, i^{\prime} \notin 2 \mathbb{N}\right)$. For any $(\mathbf{i}, u): \mathbf{p}_{+}$, the monomial $\left[y_{\mathbf{i}}(u)\right]_{\mathbf{T}}$ is positive for $u=-\frac{1}{2} h^{\vee},-\frac{1}{2} h^{\vee}-\frac{1}{2}$ and negative otherwise.

(iii) $y_{i i^{\prime}}(\ell)$ equals $y_{i, 2 \ell-i^{\prime}}^{-1}$ if $i \leq r-1$, and $y_{i, \ell-i^{\prime}}^{-1}$ if $i=r, r+1$.

(iv) For even $r, y_{i i^{\prime}}\left(-h^{\vee}\right)$ equals $y_{i i^{\prime}}^{-1}$ if $i \leq r-1$, and $y_{2 r+1-i, i^{\prime}}^{-1}$ if $i=r, r+1$.

For odd $r, y_{i i^{\prime}}\left(-h^{\vee}\right)=y_{i i^{\prime}}^{-1}$.

We obtain two important corollaries of Propositions 3.10 and 3.16.

Theorem 3.17. For $\left[\mathcal{G}_{Y}(B, y)\right]_{\mathbf{T}}$ the following relations hold:

(i) Half periodicity: $\left[y_{\mathbf{i}}\left(u+h^{\vee}+\ell\right)\right]_{\mathbf{T}}=\left[y_{\boldsymbol{\omega}(\mathbf{i})}(u)\right]_{\mathbf{T}}$.

(ii) Full periodicity: $\left[y_{\mathbf{i}}\left(u+2\left(h^{\vee}+\ell\right)\right)\right]_{\mathbf{T}}=\left[y_{\mathbf{i}}(u)\right]_{\mathbf{T}}$. 
Theorem 3.18. For $\left[\mathcal{G}_{Y}(B, y)\right]_{\mathbf{T}}$, let $N_{+}$and $N_{-}$denote the total numbers of the positive and negative monomials, respectively, among $\left[y_{\mathbf{i}}(u)\right]_{\mathbf{T}}$ for $(\mathbf{i}, u): \mathbf{p}_{+}$in the region $0 \leq u<2\left(h^{\vee}+\ell\right)$. Then

$$
N_{+}=2 \ell(2 r \ell-\ell-1), \quad N_{-}=2 r(2 \ell r-r-1) .
$$

We observe the symmetry (the level-rank duality) for the numbers $N_{+}$and $N_{-}$ under the exchange of $r$ and $\ell$.

\section{§3.8. Periodicities and dilogarithm identities}

Applying [IIKKN, Theorem 5.1] to Theorem 3.17, we obtain the periodicities:

Theorem 3.19. For $\mathcal{A}(B, x, y)$, the following relations hold:

(i) Half periodicity: $x_{\mathbf{i}}\left(u+h^{\vee}+\ell\right)=x_{\boldsymbol{\omega}(\mathbf{i})}(u)$.

(ii) Full periodicity: $x_{\mathbf{i}}\left(u+2\left(h^{\vee}+\ell\right)\right)=x_{\mathbf{i}}(u)$.

Theorem 3.20. For $\mathcal{G}(B, y)$, the following relations hold:

(i) Half periodicity: $y_{\mathbf{i}}\left(u+h^{\vee}+\ell\right)=y_{\boldsymbol{\omega}(\mathbf{i})}(u)$.

(ii) Full periodicity: $y_{\mathbf{i}}\left(u+2\left(h^{\vee}+\ell\right)\right)=y_{\mathbf{i}}(u)$.

Theorems 2.5 and 2.6 for $C_{r}$ follow from Theorems 3.6, 3.9, 3.19, and 3.20. Furthermore, Theorem 2.10 for $C_{r}$ is obtained from the above periodicities and Theorem 3.18 as in the $B_{r}$ case [IIKKN, Section 6].

\section{§4. Type $F_{4}$}

The $F_{4}$ case is quite parallel to the $B_{r}$ and $C_{r}$ cases. We do not repeat the same definitions unless otherwise mentioned. Again, the properties of the tropical Ysystem at level 2 (Proposition 4.7) are crucial and specific to $F_{4}$.

\section{§4.1. Parity decompositions of T-systems and Y-systems}

For a triplet $(a, m, u) \in \mathcal{I}_{\ell}$, we reset the 'parity conditions' $\mathbf{P}_{+}$and $\mathbf{P}_{-}$to be

$$
\begin{aligned}
& \mathbf{P}_{+}: 2 u \text { is even if } a=1,2 ; a+m+2 u \text { is odd if } a=3,4 \\
& \mathbf{P}_{-}: 2 u \text { is odd if } a=1,2 ; a+m+2 u \text { is even if } a=3,4 .
\end{aligned}
$$

Then we have $\mathcal{T}_{\ell}^{\circ}\left(F_{4}\right)_{+} \simeq \mathcal{T}_{\ell}^{\circ}\left(F_{4}\right)_{-}$via $T_{m}^{(a)}(u) \mapsto T_{m}^{(a)}\left(u+\frac{1}{2}\right)$ and

$$
\mathcal{T}_{\ell}^{\circ}\left(F_{4}\right) \simeq \mathcal{T}_{\ell}^{\circ}\left(F_{4}\right)_{+} \otimes_{\mathbb{Z}} \mathcal{T}_{\ell}^{\circ}\left(F_{4}\right)_{-} .
$$


For a triplet $(a, m, u) \in \mathcal{I}_{\ell}$, we reset the parity conditions $\mathbf{P}_{+}^{\prime}$ and $\mathbf{P}_{-}^{\prime}$ to be

$$
\begin{aligned}
& \mathbf{P}_{+}^{\prime}: 2 u \text { is even if } a=1,2 ; a+m+2 u \text { is even if } a=3,4 \\
& \mathbf{P}_{-}^{\prime}: 2 u \text { is odd if } a=1,2 ; a+m+2 u \text { is odd if } a=3,4 .
\end{aligned}
$$

We have

$$
(a, m, u): \mathbf{P}_{+}^{\prime} \Leftrightarrow\left(a, m, u \pm \frac{1}{t_{a}}\right): \mathbf{P}_{+}
$$

Also, we have $y_{\ell}^{\circ}\left(F_{4}\right)_{+} \simeq y_{\ell}^{\circ}\left(F_{4}\right)_{-}$via $Y_{m}^{(a)}(u) \mapsto Y_{m}^{(a)}\left(u+\frac{1}{2}\right), 1+Y_{m}^{(a)}(u) \mapsto$ $1+Y_{m}^{(a)}\left(u+\frac{1}{2}\right)$, and

$$
y_{\ell}^{\circ}\left(F_{4}\right) \simeq y_{\ell}^{\circ}\left(F_{4}\right)_{+} \times y_{\ell}^{\circ}\left(F_{4}\right)_{-} .
$$

\section{§4.2. Quiver $Q_{\ell}\left(F_{4}\right)$}

With type $F_{4}$ and $\ell \geq 2$ we associate the quiver $Q_{\ell}\left(F_{4}\right)$ by Figure 4 , where the right column in the left quiver and the middle column in the right quiver are
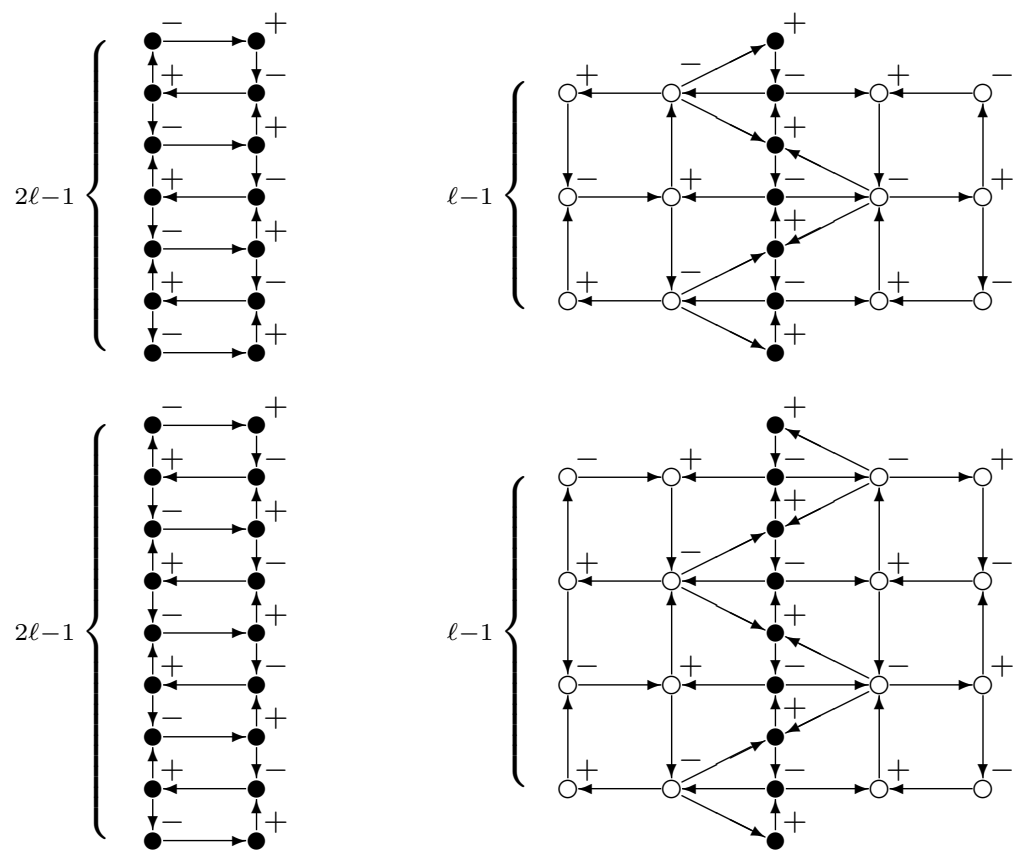

Figure 4. The quiver $Q_{\ell}\left(F_{4}\right)$ for $\ell$ even (top) and for $\ell$ odd (bottom), where we identify the right column in the left quiver with the middle column in the right quiver. 
identified. Also, we assign an empty or filled circle $\circ / \bullet$ and a sign $+/-$ to each vertex.

Let us choose the index set $\mathbf{I}$ of the vertices of $Q_{\ell}\left(F_{4}\right)$ so that $\mathbf{i}=\left(i, i^{\prime}\right) \in \mathbf{I}$ represents the vertex in the $i^{\prime}$ th row (from the bottom) and the $i$ th column (from the left) of the right quiver for $i=1,2,3$, in the $(i-1)$ th column of the right quiver for $i=5,6$, and in the left column of the left quiver for $i=4$. Thus, $i=1, \ldots, 6$, and $i^{\prime}=1, \ldots, \ell-1$ if $i=1,2,5,6$, while $i^{\prime}=1, \ldots, 2 \ell-1$ if $i=3,4$.

Let $\boldsymbol{r}$ be the involution acting on $\mathbf{I}$ by left-right reflection of the right quiver. Let $\boldsymbol{\omega}$ be the involution acting on $\mathbf{I}$ by up-down reflection of the left quiver and $180^{\circ}$ rotation of the right quiver.

Lemma 4.1. Let $Q=Q_{\ell}\left(F_{4}\right)$.

(i) We have the same periodic sequence of mutations of quivers as in (3.9).

(ii) $\boldsymbol{\omega}(Q)=Q$ if $h^{\vee}+\ell$ is even, and $\boldsymbol{\omega}(Q)=\boldsymbol{r}(Q)$ if $h^{\vee}+\ell$ is odd.

\section{$\S 4.3$. Cluster algebra and alternative labels}

Let $B_{\ell}\left(F_{4}\right)$ be the skew-symmetric matrix corresponding to the quiver $Q_{\ell}\left(F_{4}\right)$. In the rest of this section, we set $B=\left(B_{\mathbf{i j}}\right)_{\mathbf{i}, \mathbf{j} \in \mathbf{I}}=B_{\ell}\left(F_{4}\right)$ unless otherwise mentioned.

Let $\mathcal{A}(B, x, y)$ be the cluster algebra with coefficients in the universal semifield $\mathbb{Q}_{\mathrm{sf}}(y)$, and $\mathcal{G}(B, y)$ be the coefficient group associated with $\mathcal{A}(B, x, y)$.

In view of Lemma 4.1 we set $x(0)=x, y(0)=y$ and define clusters $x(u)=$ $\left(x_{\mathbf{i}}(u)\right)_{\mathbf{i} \in \mathbf{I}}\left(u \in \frac{1}{2} \mathbb{Z}\right)$ and coefficient tuples $y(u)=\left(y_{\mathbf{i}}(u)\right)_{\mathbf{i} \in \mathbf{I}}\left(u \in \frac{1}{2} \mathbb{Z}\right)$ by the sequence of mutations (3.11).

For a pair $(\mathbf{i}, u) \in \mathbf{I} \times \frac{1}{2} \mathbb{Z}$, we set the same parity condition $\mathbf{p}_{+}$and $\mathbf{p}_{-}$as in (3.12). We have (3.13), and each $(\mathbf{i}, u): \mathbf{p}_{+}$is a mutation point of (3.11) in the forward direction of $u$, while each $(\mathbf{i}, u): \mathbf{p}_{-}$is one in the backward direction of $u$ as before.

Lemma 4.2. Below $\equiv$ means equivalence modulo $2 \mathbb{Z}$.

(i) The map $g: \mathcal{I}_{\ell+} \rightarrow\left\{(\mathbf{i}, u): \mathbf{p}_{+}\right\}$defined by

$$
\left(a, m, u-\frac{1}{t_{a}}\right) \mapsto \begin{cases}((a, m), u), & a=1,2 ; a+m+u \equiv 0, \\ ((7-a, m), u), & a=1,2 ; a+m+u \equiv 1, \\ ((a, m), u), & a=3,4,\end{cases}
$$

is a bijection. 


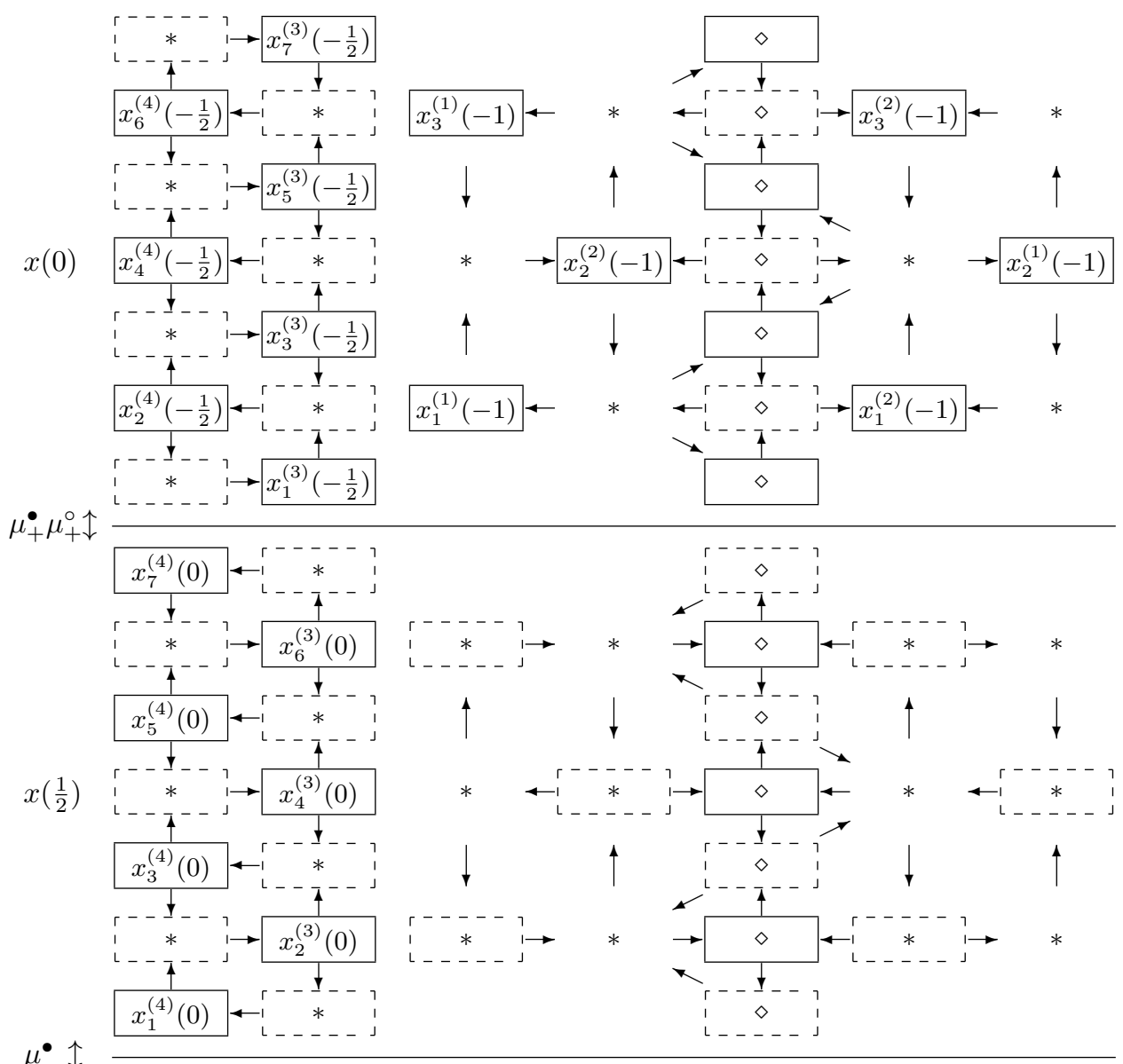

Figure 5. (Continued in Figure 6.) Labeling of cluster variables $x_{\mathbf{i}}(u)$ by $\mathcal{I}_{\ell+}$ for $F_{4}$, $\ell=4$. The middle column in the right quiver (marked by $\diamond$ ) is identified with the right column in the left quiver.

(ii) The map $g^{\prime}: \mathcal{I}_{\ell+}^{\prime} \rightarrow\left\{(\mathbf{i}, u): \mathbf{p}_{+}\right\}$defined by

$$
(a, m, u) \mapsto \begin{cases}((a, m), u), & a=1,2 ; a+m+u \equiv 0, \\ ((7-a, m), u), & a=1,2 ; a+m+u \equiv 1, \\ ((a, m), u), & a=3,4,\end{cases}
$$

is a bijection. 


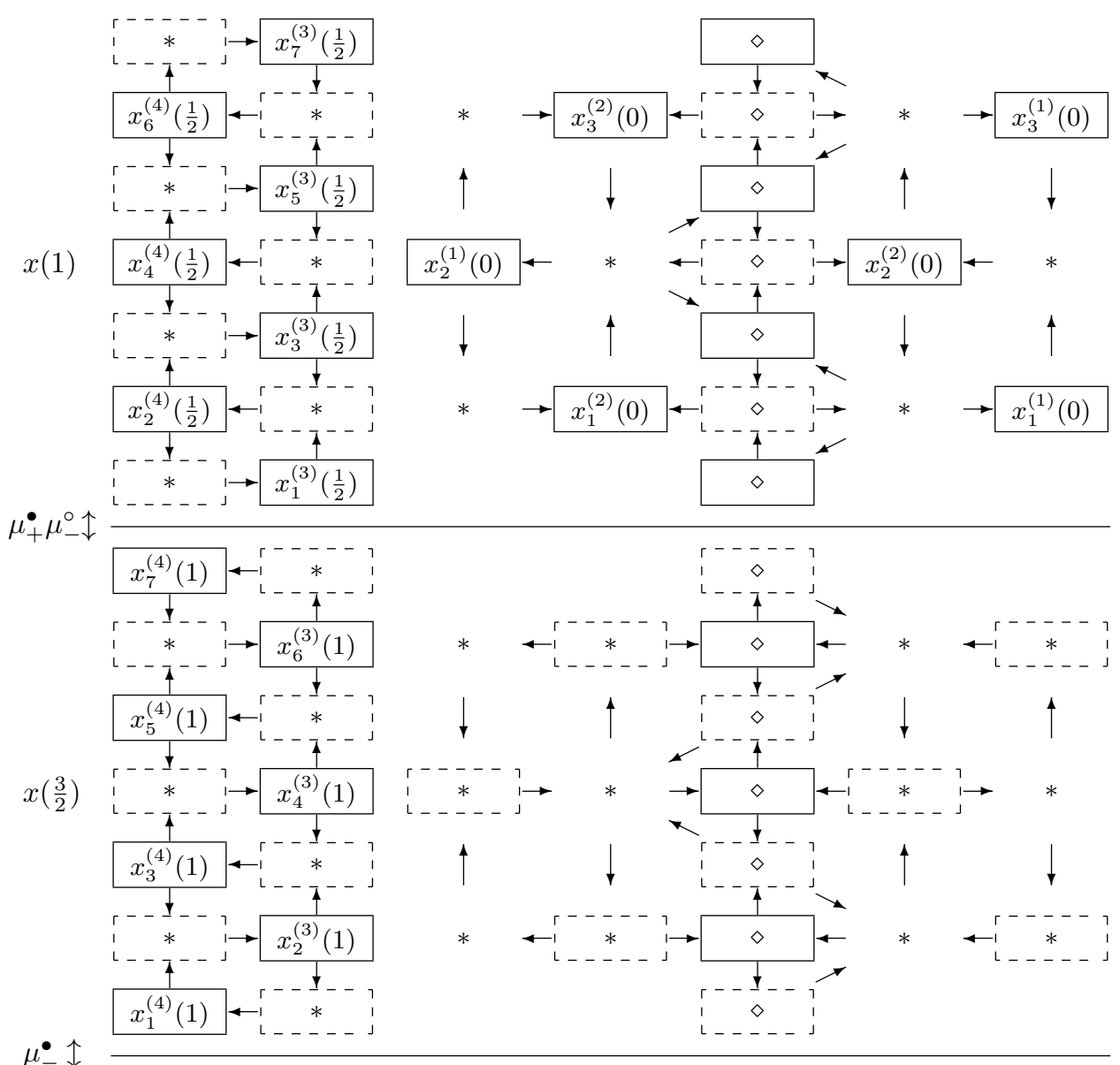

Figure 6. Continuation of Figure 5.

We introduce alternative labels $x_{\mathbf{i}}(u)=x_{m}^{(a)}\left(u-1 / t_{a}\right)\left(\left(a, m, u-1 / t_{a}\right) \in \mathcal{I}_{\ell+}\right)$ for $(\mathbf{i}, u)=g\left(\left(a, m, u-1 / t_{a}\right)\right)$ and $y_{\mathbf{i}}(u)=y_{m}^{(a)}(u)\left((a, m, u) \in \mathcal{I}_{\ell+}^{\prime}\right)$ for $(\mathbf{i}, u)=$ $g^{\prime}((a, m, u))$, respectively. See Figures 5-6.

\section{$\S 4.4$. T-system and cluster algebra}

The result in this subsection is completely parallel to the $B_{r}$ and $C_{r}$ cases.

Lemma 4.3. The family $\left\{x_{m}^{(a)}(u) \mid(a, m, u) \in \mathcal{I}_{\ell+}\right\}$ satisfies the system of relations (3.16) with $G(b, k, v ; a, m, u)$ for $\mathbb{T}_{\ell}\left(F_{4}\right)$. In particular, the family $\left\{\left[x_{m}^{(a)}(u)\right]_{\mathbf{1}} \mid\right.$ $\left.(a, m, u) \in \mathcal{I}_{\ell+}\right\}$ satisfies the T-system $\mathbb{T}_{\ell}\left(F_{4}\right)$ in $\mathcal{A}(B, x)$ after replacing $T_{m}^{(a)}(u)$ with $\left[x_{m}^{(a)}(u)\right]_{\mathbf{1}}$. 
The T-subalgebra $\mathcal{A}_{T}(B, x)$ is defined as in Definition 3.5.

Theorem 4.4. The ring $\mathcal{T}_{\ell}^{\circ}\left(F_{4}\right)_{+}$is isomorphic to $\mathcal{A}_{T}(B, x)$ via the correspondence $T_{m}^{(a)}(u) \mapsto\left[x_{m}^{(a)}(u)\right]_{1}$.

\section{$\S 4.5$. Y-system and cluster algebra}

The result in this subsection is completely parallel to the $B_{r}$ and $C_{r}$ cases.

Lemma 4.5. The family $\left\{y_{m}^{(a)}(u) \mid(a, m, u) \in \mathcal{I}_{\ell+}^{\prime}\right\}$ satisfies the $Y$-system $\mathbb{Y}_{\ell}\left(F_{4}\right)$ after replacing $Y_{m}^{(a)}(u)$ with $y_{m}^{(a)}(u)$.

The Y-subgroup $\mathcal{G}_{Y}(B, y)$ is defined as in Definition 3.8.

Theorem 4.6. The group $y_{\ell}\left(F_{4}\right)_{+}$is isomorphic to $\mathcal{G}_{Y}(B, y)$ via the correspondence $Y_{m}^{(a)}(u) \mapsto y_{m}^{(a)}(u)$ and $1+Y_{m}^{(a)}(u) \mapsto 1+y_{m}^{(a)}(u)$.

\section{§4.6. Tropical Y-system at level 2}

By direct computations, the following properties are verified.

Proposition 4.7. For $\left[\mathcal{G}_{Y}(B, y)\right]_{\mathbf{T}}$ with $B=B_{2}\left(F_{4}\right)$, the following facts hold:

(i) Let $0 \leq u<2$. For any $(\mathbf{i}, u)$ : $\mathbf{p}_{+}$, the monomial $\left[y_{\mathbf{i}}(u)\right]_{\mathbf{T}}$ is positive.

(ii) Let $-h^{\vee} \leq u<0$.

(a) Let $\mathbf{i}=(1,1),(2,1),(5,1),(6,1),(3,2)$, or $(4,2)$. For any $(\mathbf{i}, u): \mathbf{p}_{+}$, the monomial $\left[y_{\mathbf{i}}(u)\right]_{\mathbf{T}}$ is negative.

(b) Let $\mathbf{i}=(3,1),(3,3),(4,1)$, or $(4,3)$. For any $(\mathbf{i}, u): \mathbf{p}_{+}$, the monomial $\left[y_{\mathbf{i}}(u)\right]_{\mathbf{T}}$ is negative for $u=-\frac{1}{2},-1,-\frac{3}{2},-3,-\frac{7}{2},-4,-\frac{11}{2},-6,-\frac{13}{2},-8$, $-\frac{17}{2},-9$ and positive for $u=-2,-\frac{5}{2},-\frac{9}{2},-5,-7,-\frac{15}{2}$.

(iii) $y_{i i^{\prime}}(2)$ equals $y_{i i^{\prime}}^{-1}$ if $i=1,2,5,6$, and $y_{i, 4-i^{\prime}}^{-1}$ if $i=3,4$.

(iv) $y_{i i^{\prime}}\left(-h^{\vee}\right)$ equals $y_{7-i, i^{\prime}}^{-1}$ if $i=1,2,5,6$, and $y_{i i^{\prime}}^{-1}$ if $i=3,4$.

Also we have a description of the 'core part' of $\left[y_{\mathbf{i}}(u)\right]_{\mathbf{T}}$ for $-h^{\vee} \leq u<0$, corresponding to the $D$ part for $C_{r}$, in terms of the root system of $E_{6}$. We use the following indexing of the Dynkin diagram $E_{6}$ :

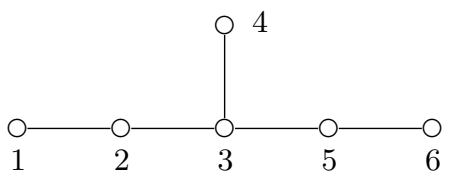


R. INOUE ET AL.

Let $\Pi=\left\{\alpha_{1}, \ldots, \alpha_{6}\right\},-\Pi, \Phi_{+}$be the sets of simple roots, of negative simple roots, and of positive roots, respectively, of type $E_{6}$. Let $\sigma_{i}$ be the piecewiselinear analogue of the simple reflection $s_{i}$, acting on the set $\Phi_{\geq-1}=\Phi_{+} \sqcup(-\Pi)$ of almost positive roots. We write $\sum_{i} m_{i} \alpha_{i} \in \Phi_{+}$as $\left[1^{m_{1}}, 2^{m_{2}}, \ldots, 6^{m_{6}}\right]$; furthermore, $\left[1^{0}, 2^{1}, 3^{1}, 4^{1}, 5^{0}, 6^{0}\right]$, for example, is abbreviated as $[2,3,4]$.

We define

$$
\sigma=\sigma_{3}\left(\sigma_{4} \sigma_{2} \sigma_{6}\right) \sigma_{3}\left(\sigma_{4} \sigma_{1} \sigma_{5}\right)
$$

Lemma 4.8. The orbits under $\sigma$ are:

$$
\begin{aligned}
-\alpha_{1} \rightarrow[1,2,3] \rightarrow[2,3,4,5,6] \rightarrow\left[1,2,3^{2}, 4,5\right] \rightarrow[5,6] \rightarrow-\alpha_{6}, \\
-\alpha_{2} \rightarrow[2,3] \rightarrow\left[1,2^{2}, 3^{2}, 4,5,6\right] \rightarrow\left[1,2^{2}, 3^{3}, 4^{2}, 5^{2}, 6\right] \\
\rightarrow\left[1,2,3^{2}, 4,5^{2}, 6\right] \rightarrow[5] \rightarrow-\alpha_{5}, \\
-\alpha_{3} \rightarrow[2,3,4] \rightarrow\left[1,2,3^{2}, 4,5,6\right] \rightarrow\left[2,3^{2}, 4,5^{2}, 6\right] \rightarrow[1,2,3,5] \rightarrow-\alpha_{3}, \\
\alpha_{3} \rightarrow[2,3,5,6] \rightarrow\left[1,2^{2}, 3^{2}, 4,5\right] \rightarrow[1,2,3,4,5,6] \rightarrow[3,4,5] \rightarrow \alpha_{3}, \\
-\alpha_{4} \rightarrow[2] \rightarrow[1,2,3,4] \rightarrow[3,4,5,6] \rightarrow[3,5] \rightarrow-\alpha_{4}, \\
\alpha_{4} \rightarrow[3,4] \rightarrow[3,5,6] \rightarrow[2,3,5] \rightarrow[1,2] \rightarrow \alpha_{4}, \\
-\alpha_{5} \rightarrow\left[2,3^{2}, 4,5,6\right] \rightarrow\left[1,2^{2}, 3^{3}, 4,5^{2}, 6\right] \rightarrow\left[1,2^{2}, 3^{2}, 4,5^{2}, 6\right] \\
\rightarrow[1,2,3,4,5] \rightarrow-\alpha_{2}, \\
-\alpha_{6} \rightarrow[6] \rightarrow\left[2,3^{2}, 4,5\right] \rightarrow[1,2,3,5,6] \rightarrow[2,3,4,5] \rightarrow[1] \rightarrow-\alpha_{1} .
\end{aligned}
$$

In particular, these elements of $\Phi_{+}$exhaust the set $\Phi_{+}$, thereby providing the orbit decomposition of $\Phi_{+}$under $\sigma$.

For $-h^{\vee} \leq u<0$, define

$$
\alpha_{i}(u)= \begin{cases}\sigma^{-u / 2}\left(-\alpha_{i}\right), & i=1,4,5 ; u \equiv 0, \\ \sigma^{-(u-1) / 2}\left(-\alpha_{i}\right), & i=2,6 ; u \equiv-1, \\ \sigma^{-(u-1) / 2}\left(\alpha_{4}\right), & i=4 ; u \equiv-1, \\ \sigma^{-(2 u-1) / 4}\left(-\alpha_{3}\right), & i=3 ; u \equiv-\frac{3}{2}, \\ \sigma^{-(2 u+1) / 4}\left(\alpha_{3}\right), & i=3 ; u \equiv-\frac{1}{2},\end{cases}
$$

where $\equiv$ is equivalence $\bmod 2 \mathbb{Z}$. By Lemma 4.8 they are (all the) positive roots of $E_{6}$.

For a monomial $m$ in $y=\left(y_{\mathbf{i}}\right)_{\mathbf{i} \in \mathbf{I}}$, let $\pi_{A}(m)$ denote the specialization with $y_{31}=y_{33}=y_{41}=y_{43}=1$. For simplicity, we set $y_{i 1}=y_{i}(i=1,2,5,6), y_{i 2}=y_{i}$ $(i=3,4)$, and also $y_{i 1}(u)=y_{i}(u)(i=1,2,5,6), y_{i 2}(u)=y_{i}(u)(i=3,4)$. We 
define the vectors $\mathbf{t}_{i}(u)=\left(t_{i}(u)_{k}\right)_{k=1}^{6}$ by

$$
\pi_{A}\left(\left[y_{i}(u)\right]_{\mathbf{T}}\right)=\prod_{k=1}^{6} y_{k}^{t_{i}(u)_{k}} .
$$

We also identify each vector $\mathbf{t}_{i}(u)$ with $\alpha=\sum_{k=1}^{6} t_{i}(u)_{k} \alpha_{k} \in \mathbb{Z} \Pi$.

Proposition 4.9. Let $-h^{\vee} \leq u<0$. Then

$$
\mathbf{t}_{i}(u)=-\alpha_{i}(u)
$$

for $(i, u)$ as in (4.12).

\section{§4.7. Tropical Y-systems at higher levels}

Due to the factorization property, we obtain the following.

Proposition 4.10. Let $\ell>2$ be an integer. For $\left[\mathcal{G}_{Y}(B, y)\right]_{\mathbf{T}}$ with $B=B_{\ell}\left(F_{4}\right)$, the following facts hold.

(i) Let $0 \leq u<\ell$. For any $(\mathbf{i}, u): \mathbf{p}_{+}$, the monomial $\left[y_{\mathbf{i}}(u)\right]_{\mathbf{T}}$ is positive.

(ii) Let $-h^{\vee} \leq u<0$.

(a) Let $\mathbf{i} \in \mathbf{I}^{\circ}$ or $\mathbf{i}=\left(3, i^{\prime}\right),\left(4, i^{\prime}\right)\left(i^{\prime} \in 2 \mathbb{N}\right)$. For any $(\mathbf{i}, u): \mathbf{p}_{+}$, the monomial $\left[y_{\mathbf{i}}(u)\right]_{\mathbf{T}}$ is negative.

(b) Let $\mathbf{i}=\left(3, i^{\prime}\right),\left(4, i^{\prime}\right)\left(i^{\prime} \notin 2 \mathbb{N}\right)$. For any $(\mathbf{i}, u): \mathbf{p}_{+}$, the monomial $\left[y_{\mathbf{i}}(u)\right]_{\mathbf{T}}$ is negative for $u=-\frac{1}{2},-1,-\frac{3}{2},-3,-\frac{7}{2},-4,-\frac{11}{2},-6,-\frac{13}{2},-8,-\frac{17}{2},-9$ and positive for $u=-2,-\frac{5}{2},-\frac{9}{2},-5,-7,-\frac{15}{2}$.

(iii) $y_{i i^{\prime}}(\ell)$ equals $y_{i, \ell-i^{\prime}}^{-1}$ if $i=1,2,5,6$, and $y_{i, 2 \ell-i^{\prime}}^{-1}$ if $i=3,4$.

(iv) $y_{i i^{\prime}}\left(-h^{\vee}\right)$ equals $y_{7-i, i^{\prime}}^{-1}$ if $i=1,2,5,6$, and $y_{i i^{\prime}}^{-1}$ if $i=3,4$.

We obtain corollaries of Propositions 4.7 and 4.10.

Theorem 4.11. For $\left[\mathcal{G}_{Y}(B, y)\right]_{\mathbf{T}}$ the following relations hold:

(i) Half periodicity: $\left[y_{\mathbf{i}}\left(u+h^{\vee}+\ell\right)\right]_{\mathbf{T}}=\left[y_{\boldsymbol{\omega}(\mathbf{i})}(u)\right]_{\mathbf{T}}$.

(ii) Full periodicity: $\left[y_{\mathbf{i}}\left(u+2\left(h^{\vee}+\ell\right)\right)\right]_{\mathbf{T}}=\left[y_{\mathbf{i}}(u)\right]_{\mathbf{T}}$.

Theorem 4.12. For $\left[\mathcal{G}_{Y}(B, y)\right]_{\mathbf{T}}$, let $N_{+}$and $N_{-}$denote the total numbers of the positive and negative monomials, respectively, among $\left[y_{\mathbf{i}}(u)\right]_{\mathbf{T}}$ for $(\mathbf{i}, u): \mathbf{p}_{+}$in the region $0 \leq u<2\left(h^{\vee}+\ell\right)$. Then

$$
N_{+}=4 \ell(3 \ell+1), \quad N_{-}=24(4 \ell-3) .
$$


R. INOUE ET AL.

\section{§4.8. Periodicities and dilogarithm identities}

Applying [IIKKN, Theorem 5.1] to Theorem 4.11, we obtain the periodicities:

Theorem 4.13. For $\mathcal{A}(B, x, y)$, the following relations hold:

(i) Half periodicity: $x_{\mathbf{i}}\left(u+h^{\vee}+\ell\right)=x_{\boldsymbol{\omega}(\mathbf{i})}(u)$.

(ii) Full periodicity: $x_{\mathbf{i}}\left(u+2\left(h^{\vee}+\ell\right)\right)=x_{\mathbf{i}}(u)$.

Theorem 4.14. For $\mathcal{G}(B, y)$, the following relations hold:

(i) Half periodicity: $y_{\mathbf{i}}\left(u+h^{\vee}+\ell\right)=y_{\boldsymbol{\omega}(\mathbf{i})}(u)$.

(ii) Full periodicity: $y_{\mathbf{i}}\left(u+2\left(h^{\vee}+\ell\right)\right)=y_{\mathbf{i}}(u)$.

Theorems 2.5 and 2.6 for $F_{4}$ follow from Theorems 4.4, 4.6, 4.13, and 4.14. Furthermore, Theorem 2.10 for $F_{4}$ is obtained from the above periodicities and Theorem 4.12 as in the $B_{r}$ case [IIKKN, Section 6].

\section{$\S 5$. Type $G_{2}$}

The $G_{2}$ case is mostly parallel to the former cases, but slightly different because the number $t$ in (2.2) is three. Again, the properties of the tropical Y-system at level 2 (Proposition 5.9) are crucial and specific to $G_{2}$.

\section{§5.1. Parity decompositions of T-systems and Y-systems}

For a triplet $(a, m, u) \in \mathcal{I}_{\ell}$, we reset the parity conditions $\mathbf{P}_{+}$and $\mathbf{P}_{-}$to be

$$
\begin{aligned}
& \mathbf{P}_{+}: a+m+3 u \text { is even, } \\
& \mathbf{P}_{-}: a+m+3 u \text { is odd. }
\end{aligned}
$$

Then we have $\mathcal{T}_{\ell}^{\circ}\left(G_{2}\right)_{+} \simeq \mathcal{T}_{\ell}^{\circ}\left(G_{2}\right)_{-}$via $T_{m}^{(a)}(u) \mapsto T_{m}^{(a)}\left(u+\frac{1}{3}\right)$ and

$$
\mathcal{T}_{\ell}^{\circ}\left(G_{2}\right) \simeq \mathcal{T}_{\ell}^{\circ}\left(G_{2}\right)_{+} \otimes_{\mathbb{Z}} \mathcal{T}_{\ell}^{\circ}\left(G_{2}\right)_{-} .
$$

For $(a, m, u) \in \mathcal{I}_{\ell}$, we reset the parity conditions $\mathbf{P}_{+}^{\prime}$ and $\mathbf{P}_{-}^{\prime}$ to be

$$
\begin{aligned}
& \mathbf{P}_{+}^{\prime}: a+m+3 u \text { is odd, } \\
& \mathbf{P}_{-}^{\prime}: a+m+3 u \text { is even. }
\end{aligned}
$$

We have

$$
(a, m, u): \mathbf{P}_{+}^{\prime} \Leftrightarrow\left(a, m, u \pm \frac{1}{t_{a}}\right): \mathbf{P}_{+}
$$


Also, we have $y_{\ell}^{\circ}\left(G_{2}\right)_{+} \simeq y_{\ell}^{\circ}\left(G_{2}\right)_{-}$via $Y_{m}^{(a)}(u) \mapsto Y_{m}^{(a)}\left(u+\frac{1}{3}\right), 1+Y_{m}^{(a)}(u) \mapsto$ $1+Y_{m}^{(a)}\left(u+\frac{1}{3}\right)$, and

$$
y_{\ell}^{\circ}\left(G_{2}\right) \simeq y_{\ell}^{\circ}\left(G_{2}\right)_{+} \times y_{\ell}^{\circ}\left(G_{2}\right)_{-} .
$$

\section{§5.2. Quiver $Q_{\ell}\left(G_{2}\right)$}

With type $G_{2}$ and $\ell \geq 2$ we associate the quiver $Q_{\ell}\left(G_{2}\right)$ by Figure 7 , where the right columns in the three quivers are identified. Also we assign an empty or filled
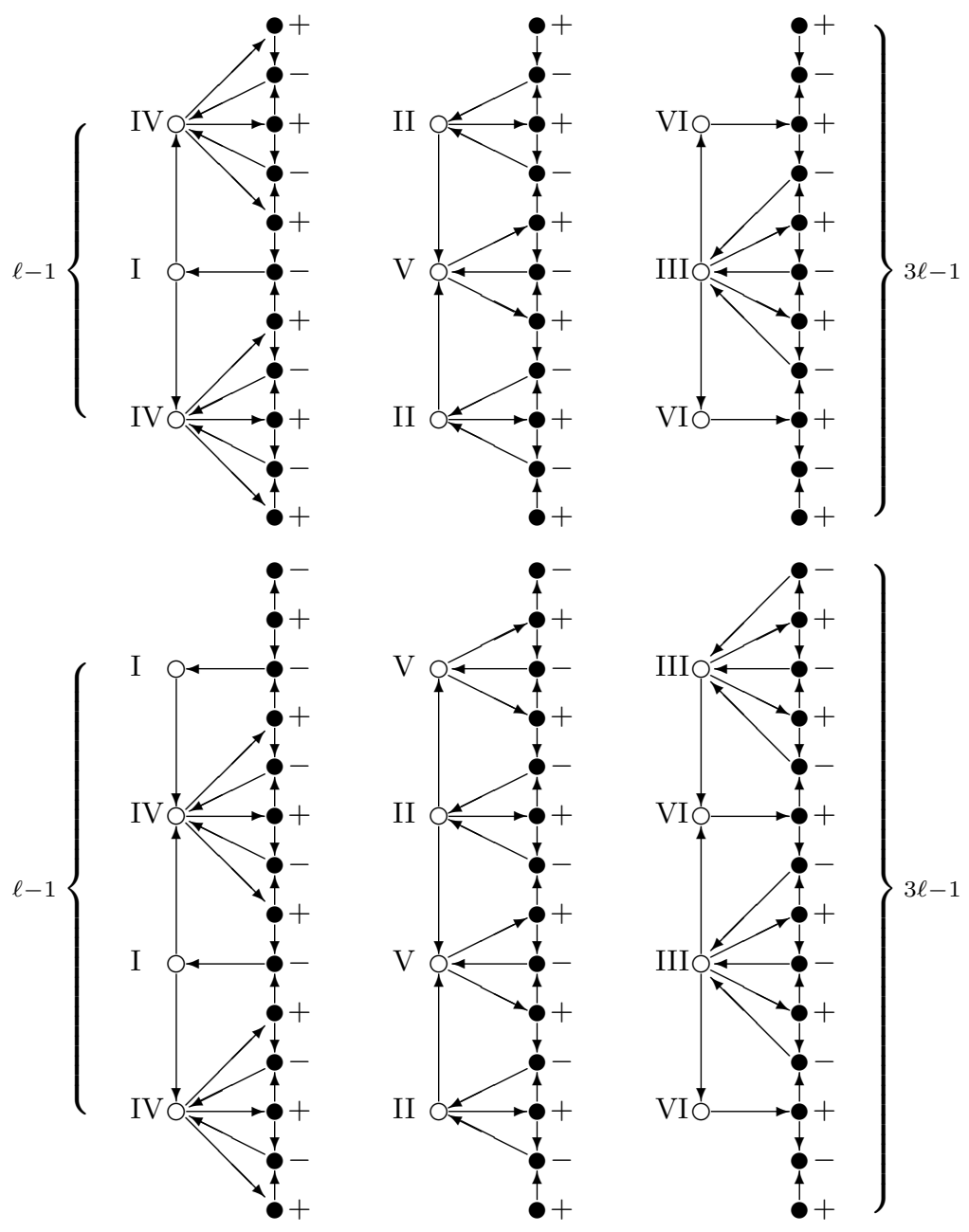

Figure 7. The quiver $Q_{\ell}\left(G_{2}\right)$ for $\ell$ even (top) and for $\ell$ odd (bottom), where we identify the right columns in the three quivers. 
circle $\circ / \bullet$ to each vertex; furthermore, we assign a sign $+/-$ to each vertex with $\bullet$ and one of the numbers I, .., VI to each vertex with $\circ$.

Let us choose the index set $\mathbf{I}$ of the vertices of $Q_{\ell}\left(G_{2}\right)$ so that $\mathbf{i}=\left(i, i^{\prime}\right) \in \mathbf{I}$ represents the vertex in the $i^{\prime}$ th row (from the bottom) and in the left column in the $i$ th quiver (from the left) for $i=1,2,3$, and in the right column in any quiver for $i=4$. Thus, $i=1, \ldots, 4$, and $i^{\prime}=1, \ldots, \ell-1$ if $i \neq 4$, while $i^{\prime}=1, \ldots, 3 \ell-1$ if $i=4$.

For a permutation $s$ of $\{1,2,3\}$, let $\boldsymbol{\nu}_{s}$ be the permutation of $\mathbf{I}$ such that $\boldsymbol{\nu}_{s}\left(i, i^{\prime}\right)$ equals $\left(s(i), i^{\prime}\right)$ for $i \neq 4$, and $\left(4, i^{\prime}\right)$ for $i=4$. Let $\boldsymbol{\omega}$ be the involution acting on $\mathbf{I}$ by up-down reflection. Let $\boldsymbol{\nu}_{s}\left(Q_{\ell}\left(G_{2}\right)\right)$ and $\boldsymbol{\omega}\left(Q_{\ell}\left(G_{2}\right)\right)$ denote the quivers induced from $Q_{\ell}\left(G_{2}\right)$ by $\boldsymbol{\nu}_{s}$ and $\boldsymbol{\omega}$, respectively.

Lemma 5.1. Let $Q=Q_{\ell}\left(G_{2}\right)$.

(i) We have a periodic sequence of mutations of quivers

$$
\begin{aligned}
& Q \stackrel{\mu_{+}^{\bullet} \mu_{\mathrm{I}}^{\circ}}{\longleftrightarrow} \boldsymbol{\nu}_{(23)}(Q)^{\mathrm{op}} \stackrel{\mu_{-}^{\bullet} \mu_{\mathrm{II}}^{\circ}}{\longleftrightarrow} \boldsymbol{\nu}_{(312)}(Q) \stackrel{\mu_{+}^{\bullet} \mu_{\mathrm{III}}^{\circ}}{\longleftrightarrow} \boldsymbol{\nu}_{(13)}(Q)^{\mathrm{op}} \\
& \stackrel{\mu_{-}^{\bullet} \mu_{\mathrm{IV}}^{\circ}}{\longleftrightarrow} \nu_{(231)}(Q) \stackrel{\mu_{+}^{\bullet} \mu_{\mathrm{V}}^{\circ}}{\longleftrightarrow} \nu_{(12)}(Q)^{\mathrm{op}} \stackrel{\mu_{-}^{\bullet} \mu_{\mathrm{VI}}^{\circ}}{\longleftrightarrow} Q .
\end{aligned}
$$

(ii) $\boldsymbol{\omega}(Q)=Q$ if $h^{\vee}+\ell$ is even, and $\boldsymbol{\omega}(Q)=\boldsymbol{\nu}_{(13)}(Q)^{\text {op }}$ if $h^{\vee}+\ell$ is odd.

See Figures 8-10 for an example.

\section{§5.3. Cluster algebra and alternative labels}

Let $B_{\ell}\left(G_{2}\right)$ be the skew-symmetric matrix corresponding to the quiver $Q_{\ell}\left(G_{2}\right)$. In the rest of the section, we set $B=\left(B_{\mathbf{i} \mathbf{j}}\right)_{\mathbf{i}, \mathbf{j} \in \mathbf{I}}=B_{\ell}\left(G_{2}\right)$ unless otherwise mentioned.

Let $\mathcal{A}(B, x, y)$ be the cluster algebra with coefficients in the universal semifield $\mathbb{Q}_{\mathrm{sf}}(y)$, and $\mathcal{G}(B, y)$ be the coefficient group associated with $\mathcal{A}(B, x, y)$.

In view of Lemma 5.1 we set $x(0)=x, y(0)=y$ and define clusters $x(u)=$ $\left(x_{\mathbf{i}}(u)\right)_{\mathbf{i} \in \mathbf{I}}\left(u \in \frac{1}{3} \mathbb{Z}\right)$ and coefficient tuples $y(u)=\left(y_{\mathbf{i}}(u)\right)_{\mathbf{i} \in \mathbf{I}}\left(u \in \frac{1}{3} \mathbb{Z}\right)$ by the sequence of mutations

$$
\begin{aligned}
& \cdots \stackrel{\mu_{-}^{\bullet} \mu_{\mathrm{VI}}^{\circ}}{\longleftrightarrow}(B, x(0), y(0)) \stackrel{\mu_{+}^{\bullet} \mu_{\mathrm{I}}^{\circ}}{\longleftrightarrow}\left(-\boldsymbol{\nu}_{(23)}(B), x\left(\frac{1}{3}\right), y\left(\frac{1}{3}\right)\right) \\
& \stackrel{\mu_{-}^{\bullet} \mu_{\mathrm{II}}^{\circ}}{\longleftrightarrow}\left(\boldsymbol{\nu}_{(312)}(B), x\left(\frac{2}{3}\right), y\left(\frac{2}{3}\right)\right) \stackrel{\mu_{+}^{\bullet} \mu_{\mathrm{III}}^{\circ}}{\longleftrightarrow}\left(-\boldsymbol{\nu}_{(13)}(B), x(1), y(1)\right) \\
& \stackrel{\mu_{-}^{\bullet} \mu_{\mathrm{IV}}^{\circ}}{\longleftrightarrow}\left(\boldsymbol{\nu}_{(231)}(B), x\left(\frac{4}{3}\right), y\left(\frac{4}{3}\right)\right) \stackrel{\stackrel{\mu_{+}^{\bullet} \mu_{\mathrm{V}}^{\circ}}{\longleftrightarrow}}{\leftrightarrows}\left(-\boldsymbol{\nu}_{(12)}(B), x\left(\frac{5}{3}\right), y\left(\frac{5}{3}\right)\right) \stackrel{\mu_{-}^{\bullet} \mu_{\mathrm{VI}}^{\circ}}{\longleftrightarrow} \cdots,
\end{aligned}
$$

where $\boldsymbol{\nu}_{s}(B)=B^{\prime}$ is defined by $B_{\boldsymbol{\nu}_{s}(\mathbf{i}) \boldsymbol{\nu}_{s}(\mathbf{j})}^{\prime}=B_{\mathbf{i j}}$. 


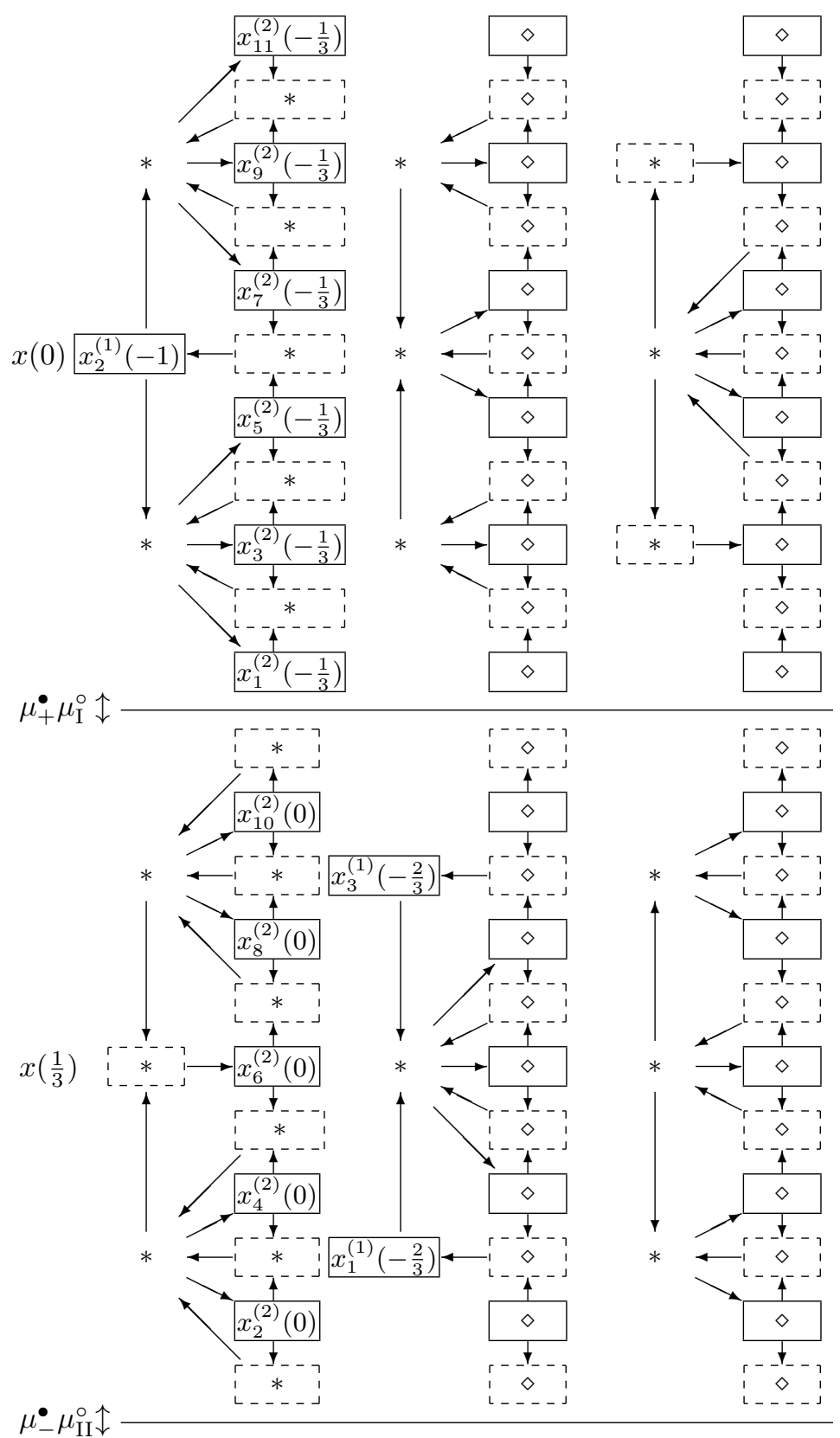

Figure 8. (Continued in Figures 9 and 10.) Labeling of cluster variables $x_{\mathbf{i}}(u)$ by $\mathcal{I}_{\ell+}$ for $G_{2}, \ell=4$. The right columns in the middle and right quivers (marked by $\diamond)$ are identified with the right column in the left quiver. 


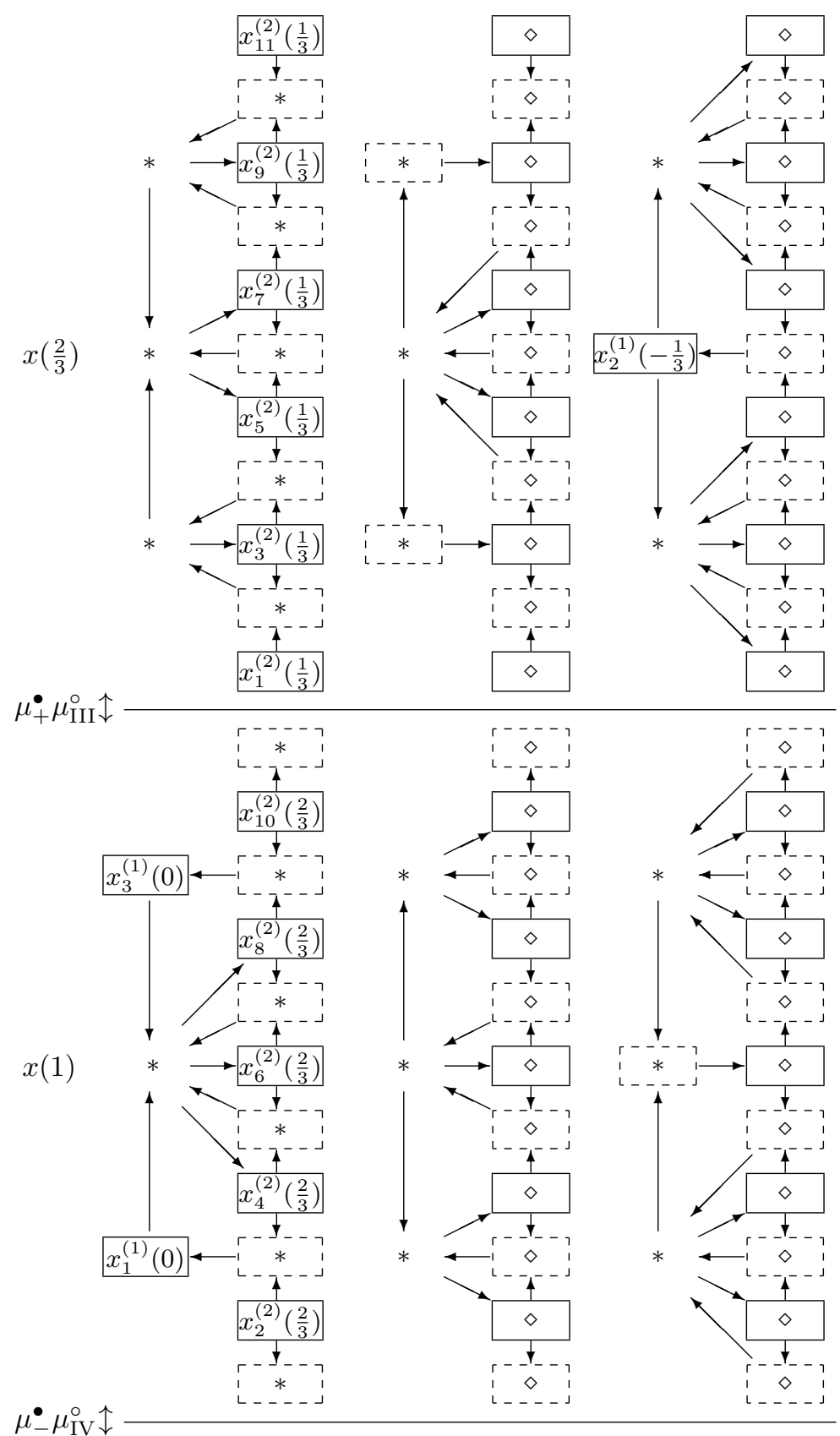

Figure 9. Continuation of Figure 8. 
Periodicities of T-systems and Y-systems II: Types $C_{r}, F_{4}$, and $G_{2}$

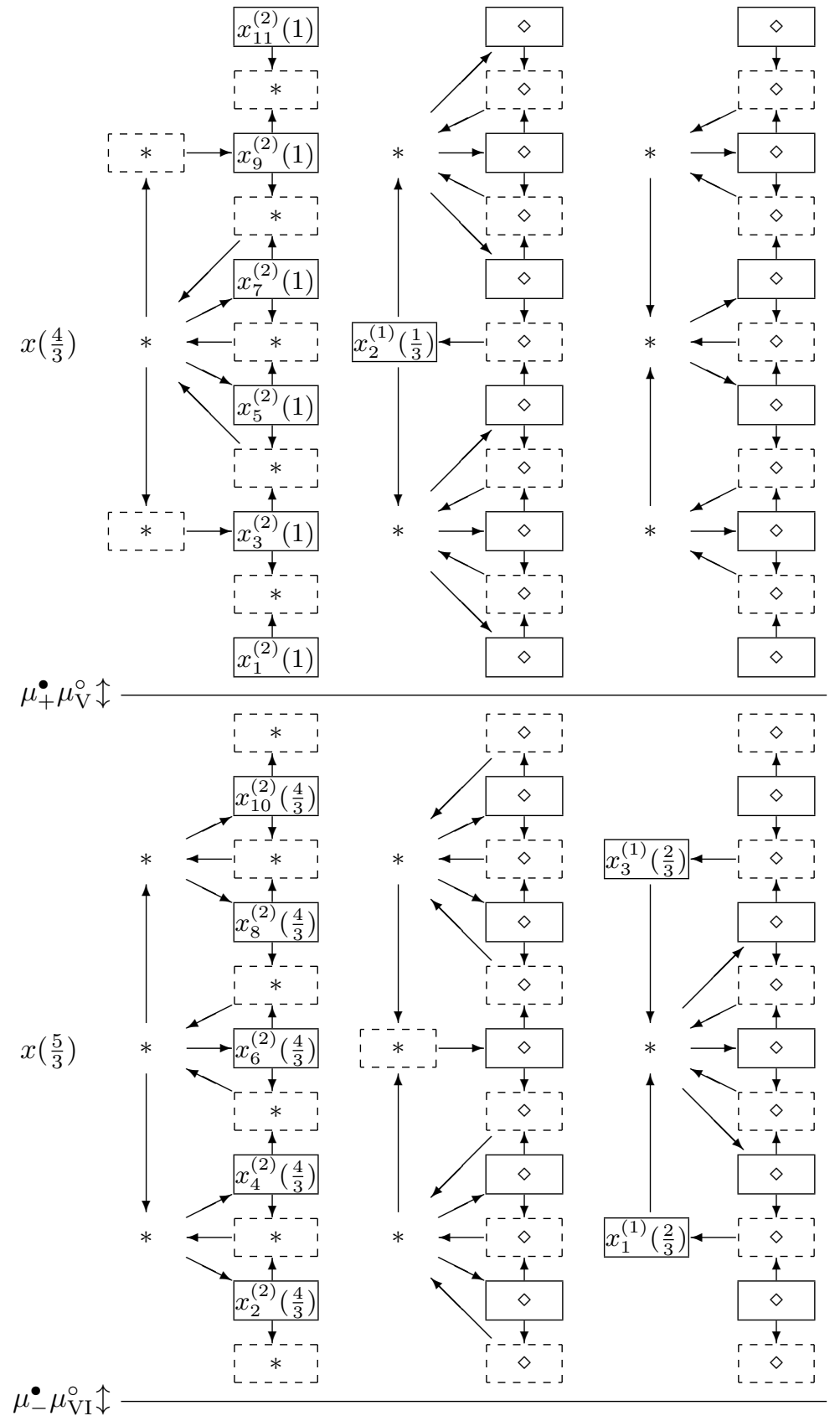

Figure 10. Continuation of Figure 9. 
For a pair $(\mathbf{i}, u) \in \mathbf{I} \times \frac{1}{3} \mathbb{Z}$, we set the parity conditions $\mathbf{p}_{+}$and $\mathbf{p}_{-}$to be

$$
\mathbf{p}_{+}:\left\{\begin{array}{ll}
\mathbf{i} \in \mathbf{I}_{\mathrm{I}}^{\circ} \sqcup \mathbf{I}_{+}^{\bullet}, & u \equiv 0, \\
\mathbf{i} \in \mathbf{I}_{\mathrm{II}}^{\circ} \sqcup \mathbf{I}_{-}^{\bullet}, & u \equiv \frac{1}{3}, \\
\mathbf{i} \in \mathbf{I}_{\mathrm{III}}^{\circ} \sqcup \mathbf{I}_{+}^{\bullet}, & u \equiv \frac{2}{3}, \\
\mathbf{i} \in \mathbf{I}_{\mathrm{IV}}^{\circ} \sqcup \mathbf{I}_{-}^{\bullet}, & u \equiv 1, \\
\mathbf{i} \in \mathbf{I}_{\mathrm{V}}^{\circ} \sqcup \mathbf{I}_{+}^{\bullet}, & u \equiv \frac{4}{3}, \\
\mathbf{i} \in \mathbf{I}_{\mathrm{VI}}^{\circ} \sqcup \mathbf{I}_{-}^{\bullet}, & u \equiv \frac{5}{3},
\end{array} \quad \mathbf{p}_{-}: \begin{cases}\mathbf{i} \in \mathbf{I}_{\mathrm{VI}}^{\circ} \sqcup \mathbf{I}_{-}^{\bullet}, & u \equiv 0, \\
\mathbf{i} \in \mathbf{I}_{\mathrm{I}}^{\circ} \sqcup \mathbf{I}_{+}^{\bullet}, & u \equiv \frac{1}{3}, \\
\mathbf{i} \in \mathbf{I}_{\mathrm{II}}^{\circ} \sqcup \mathbf{I}_{-}^{\bullet}, & u \equiv \frac{2}{3}, \\
\mathbf{i} \in \mathbf{I}_{\mathrm{III}}^{\circ} \sqcup \mathbf{I}_{+}^{\bullet}, & u \equiv 1, \\
\mathbf{i} \in \mathbf{I}_{\mathrm{IV}}^{\circ} \sqcup \mathbf{I}_{-}^{\bullet}, & u \equiv \frac{4}{3}, \\
\mathbf{i} \in \mathbf{I}_{\mathrm{V}}^{\circ} \sqcup \mathbf{I}_{+}^{\bullet}, & u \equiv \frac{5}{3},\end{cases}\right.
$$

where $\equiv$ is equivalence modulo $2 \mathbb{Z}$. We have

$$
(\mathbf{i}, u): \mathbf{p}_{+} \Leftrightarrow\left(\mathbf{i}, u+\frac{1}{3}\right): \mathbf{p}_{-} .
$$

Each $(\mathbf{i}, u): \mathbf{p}_{+}$is a mutation point of (5.9) in the forward direction of $u$, and each $(\mathbf{i}, u): \mathbf{p}_{-}$is one in the backward direction of $u$.

Lemma 5.2. Below $\equiv$ means equivalence modulo $2 \mathbb{Z}$.

(i) The map $g: \mathcal{I}_{\ell+} \rightarrow\left\{(\mathbf{i}, u): \mathbf{p}_{+}\right\}$defined by

$$
\left(a, m, u-\frac{1}{t_{a}}\right) \mapsto \begin{cases}((1, m), u), & a=1 ; m+u \equiv 0 \\ ((2, m), u), & a=1 ; m+u \equiv \frac{4}{3} \\ ((3, m), u), & a=1 ; m+u \equiv \frac{2}{3} \\ ((4, m), u), & a=2,\end{cases}
$$

is a bijection.

(ii) The map $g^{\prime}: \mathcal{I}_{\ell+}^{\prime} \rightarrow\left\{(\mathbf{i}, u): \mathbf{p}_{+}\right\}$defined by

$$
(a, m, u) \mapsto \begin{cases}((1, m), u), & a=1 ; m+u \equiv 0 \\ ((2, m), u), & a=1 ; m+u \equiv \frac{4}{3} \\ ((3, m), u), & a=1 ; m+u \equiv \frac{2}{3} \\ ((4, m), u), & a=2,\end{cases}
$$

is a bijection.

We introduce alternative labels $x_{\mathbf{i}}(u)=x_{m}^{(a)}\left(u-1 / t_{a}\right)\left(\left(a, m, u-1 / t_{a}\right) \in \mathcal{I}_{\ell+}\right)$ for $(\mathbf{i}, u)=g\left(\left(a, m, u-1 / t_{a}\right)\right)$ and $y_{\mathbf{i}}(u)=y_{m}^{(a)}(u)\left((a, m, u) \in \mathcal{I}_{\ell+}^{\prime}\right)$ for $(\mathbf{i}, u)=$ $g^{\prime}((a, m, u))$, respectively. See Figures 8-10 for an example.

\section{$\S 5.4$. T-system and cluster algebra}

Lemma 5.3. The family $\left\{x_{m}^{(a)}(u) \mid(a, m, u) \in \mathcal{I}_{\ell+}\right\}$ satisfies the system of relations (3.16) with $G(b, k, v ; a, m, u)$ for $\mathbb{T}_{\ell}\left(G_{2}\right)$. In particular, the family 
$\left\{\left[x_{m}^{(a)}(u)\right]_{1} \mid(a, m, u) \in \mathcal{I}_{\ell+}\right\}$ satisfies the T-system $\mathbb{T}_{\ell}\left(G_{2}\right)$ in $\mathcal{A}(B, x)$ after replacing $T_{m}^{(a)}(u)$ with $\left[x_{m}^{(a)}(u)\right]_{\mathbf{1}}$.

Definition 5.4. The T-subalgebra $\mathcal{A}_{T}(B, x)$ of $\mathcal{A}(B, x)$ associated with the sequence (5.9) is the subring of $\mathcal{A}(B, x)$ generated by $\left[x_{\mathbf{i}}(u)\right]_{\mathbf{1}}\left((\mathbf{i}, u) \in \mathbf{I} \times \frac{1}{3} \mathbb{Z}\right)$.

Theorem 5.5. The ring $\mathcal{T}_{\ell}^{\circ}\left(G_{2}\right)_{+}$is isomorphic to $\mathcal{A}_{T}(B, x)$ via the correspondence $T_{m}^{(a)}(u) \mapsto\left[x_{m}^{(a)}(u)\right]_{1}$.

\section{§5.5. Y-system and cluster algebra}

Lemma 5.6. The family $\left\{y_{m}^{(a)}(u) \mid(a, m, u) \in \mathcal{I}_{\ell+}^{\prime}\right\}$ satisfies the $Y$-system $\mathbb{Y}_{\ell}\left(G_{2}\right)$ after replacing $Y_{m}^{(a)}(u)$ with $y_{m}^{(a)}(u)$.

Definition 5.7. The $Y$-subgroup $\mathcal{G}_{Y}(B, y)$ of $\mathcal{G}(B, y)$ associated with the sequence (5.9) is the subgroup of $\mathcal{G}(B, y)$ generated by $y_{\mathbf{i}}(u)\left((\mathbf{i}, u) \in \mathbf{I} \times \frac{1}{3} \mathbb{Z}\right)$ and $1+y_{\mathbf{i}}(u)$ $\left((\mathbf{i}, u): \mathbf{p}_{+}\right.$or $\left.\mathbf{p}_{-}\right)$.

Theorem 5.8. The group $y_{\ell}^{\circ}\left(G_{2}\right)_{+}$is isomorphic to $\mathcal{G}_{Y}(B, y)$ via the correspondence $Y_{m}^{(a)}(u) \mapsto y_{m}^{(a)}(u)$ and $1+Y_{m}^{(a)}(u) \mapsto 1+y_{m}^{(a)}(u)$.

\section{§5.6. Tropical Y-system at level 2}

By direct computations, the following properties are verified.

Proposition 5.9. For $\left[\mathcal{G}_{Y}(B, y)\right]_{\mathbf{T}}$ with $B=B_{2}\left(G_{2}\right)$, the following facts hold:

(i) Let $0 \leq u<2$. For any $(\mathbf{i}, u)$ : $\mathbf{p}_{+}$, the monomial $\left[y_{\mathbf{i}}(u)\right]_{\mathbf{T}}$ is positive.

(ii) Let $-h^{\vee} \leq u<0$.

(a) Let $\mathbf{i}=(1,1),(2,1),(3,1)$, or $(4,3)$. For any $(\mathbf{i}, u): \mathbf{p}_{+}$, the monomial $\left[y_{\mathbf{i}}(u)\right]_{\mathbf{T}}$ is negative.

(b) Let $\mathbf{i}=(4,1),(4,2),(4,4)$, or $(4,5)$. For any $(\mathbf{i}, u): \mathbf{p}_{+}$, the monomial $\left[y_{\mathbf{i}}(u)\right]_{\mathbf{T}}$ is negative for $u=-\frac{1}{3},-\frac{2}{3},-2,-\frac{7}{3},-\frac{11}{3},-4$, and positive for $u=-1,-\frac{4}{3},-\frac{5}{3},-\frac{8}{3},-3,-\frac{10}{3}$.

(iii) $y_{i i^{\prime}}(2)$ equals $y_{i i^{\prime}}^{-1}$ if $i \neq 4$, and $y_{4,6-i^{\prime}}^{-1}$ if $i=4$.

(iv) $y_{i i^{\prime}}\left(-h^{\vee}\right)=y_{i i^{\prime}}^{-1}$.

Also we have a description of the core part of $\left[y_{\mathbf{i}}(u)\right]_{\mathbf{T}}$ in the region $-h^{\vee} \leq$ $u<0$ in terms of the root system of $D_{4}$. We use the following indexing of the 
Dynkin diagram $D_{4}$ :



Let $\Pi=\left\{\alpha_{1}, \ldots, \alpha_{4}\right\},-\Pi, \Phi_{+}$be the sets of simple roots, of negative simple roots, and of positive roots, respectively, of type $D_{4}$. Let $\sigma_{i}$ be the piecewise-linear analogue of the simple reflection $s_{i}$, acting on the set $\Phi_{\geq-1}=\Phi_{+} \sqcup(-\Pi)$ of almost positive roots. We define

$$
\sigma=\sigma_{3} \sigma_{4} \sigma_{1} \sigma_{4} \sigma_{2} \sigma_{4}
$$

Lemma 5.10. The orbits under $\sigma$ are:

$$
\begin{aligned}
-\alpha_{1} & \rightarrow \alpha_{1}+\alpha_{3}+\alpha_{4} \rightarrow \alpha_{1}+\alpha_{2}+\alpha_{4} \rightarrow-\alpha_{1}, \\
-\alpha_{2} & \rightarrow \alpha_{1}+\alpha_{2}+\alpha_{3}+\alpha_{4} \rightarrow \alpha_{2}+\alpha_{4} \rightarrow-\alpha_{2}, \\
-\alpha_{3} & \rightarrow \alpha_{3} \rightarrow \alpha_{1}+\alpha_{2}+\alpha_{3}+2 \alpha_{4} \rightarrow-\alpha_{3}, \\
-\alpha_{4} & \rightarrow \alpha_{2}+\alpha_{3}+\alpha_{4} \rightarrow \alpha_{4} \rightarrow \alpha_{1} \\
& \rightarrow \alpha_{2} \rightarrow \alpha_{3}+\alpha_{4} \rightarrow \alpha_{1}+\alpha_{4} \rightarrow-\alpha_{4} .
\end{aligned}
$$

In particular, these elements of $\Phi_{+}$exhaust the set $\Phi_{+}$, thereby providing the orbit decomposition of $\Phi_{+}$under $\sigma$.

For $-h^{\vee} \leq u<0$, define

$$
\alpha_{i}(u)= \begin{cases}\sigma^{-(u-1) / 2}\left(-\alpha_{1}\right), & i=1 ; u=-1,-3, \\ \sigma^{-(3 u-1) / 6}\left(-\alpha_{2}\right), & i=2 ; u=-\frac{5}{3},-\frac{11}{3}, \\ \sigma^{-(3 u-5) / 6}\left(-\alpha_{3}\right), & i=3 ; u=-\frac{1}{3},-\frac{7}{3}, \\ \sigma^{-(3 u+2) / 6}\left(\alpha_{3}+\alpha_{4}\right), & i=4 ; u=-\frac{2}{3},-\frac{8}{3}, \\ \sigma^{-(3 u+4) / 6}\left(\alpha_{1}\right), & i=4 ; u=-\frac{4}{3},-\frac{10}{3}, \\ \sigma^{-u / 2}\left(-\alpha_{4}\right), & i=4 ; u=-2,-4 .\end{cases}
$$

By Lemma 5.10 these are (all the) positive roots of $D_{4}$.

For a monomial $m$ in $y=\left(y_{\mathbf{i}}\right)_{\mathbf{i} \in \mathbf{I}}$, let $\pi_{D}(m)$ denote the specialization with $y_{41}=y_{42}=y_{44}=y_{45}=1$. For simplicity, we set $y_{i 1}=y_{i}(i \neq 4), y_{43}=y_{4}$, and also $y_{i 1}(u)=y_{i}(u)(i \neq 4), y_{43}(u)=y_{4}(u)$. We define the vectors $\mathbf{t}_{i}(u)=\left(t_{i}(u)_{k}\right)_{k=1}^{4}$ by

$$
\pi_{D}\left(\left[y_{i}(u)\right]_{\mathbf{T}}\right)=\prod_{k=1}^{4} y_{k}^{t_{i}(u)_{k}}
$$


We also identify each vector $\mathbf{t}_{i}(u)$ with $\alpha=\sum_{k=1}^{4} t_{i}(u)_{k} \alpha_{k} \in \mathbb{Z} \Pi$.

Proposition 5.11. Let $-h^{\vee} \leq u<0$. Then

$$
\mathbf{t}_{i}(u)=-\alpha_{i}(u)
$$

for $(i, u)$ as in (5.16).

\section{§5.7. Tropical Y-systems of higher levels}

Proposition 5.12. Let $\ell>2$ be an integer. For $\left[\mathcal{G}_{Y}(B, y)\right]_{\mathbf{T}}$ with $B=B_{\ell}\left(G_{2}\right)$, the following facts hold:

(i) Let $0 \leq u<\ell$. For any $(\mathbf{i}, u): \mathbf{p}_{+}$, the monomial $\left[y_{\mathbf{i}}(u)\right]_{\mathbf{T}}$ is positive.

(ii) Let $-h^{\vee} \leq u<0$.

(a) Let $\mathbf{i} \in \mathbf{I}^{\circ}$ or $\mathbf{i}=\left(4, i^{\prime}\right)\left(i^{\prime} \in 3 \mathbb{N}\right)$. For any $(\mathbf{i}, u): \mathbf{p}_{+}$, the monomial $\left[y_{\mathbf{i}}(u)\right]_{\mathbf{T}}$ is negative.

(b) Let $\mathbf{i}=\left(4, i^{\prime}\right)\left(i^{\prime} \notin 3 \mathbb{N}\right)$. For any $(\mathbf{i}, u): \mathbf{p}_{+}$, the monomial $\left[y_{\mathbf{i}}(u)\right]_{\mathbf{T}}$ is negative for $u=-\frac{1}{3},-\frac{2}{3},-2,-\frac{7}{3},-\frac{11}{3},-4$ and positive for $u=-1,-\frac{4}{3},-\frac{5}{3}$, $-\frac{8}{3},-3,-\frac{10}{3}$.

(iii) $y_{i i^{\prime}}(\ell)$ equals $y_{i, \ell-i^{\prime}}^{-1}$ if $i \neq 4$, and $y_{4,3 \ell-i^{\prime}}^{-1}$ if $i=4$.

(iv) $y_{i i^{\prime}}\left(-h^{\vee}\right)=y_{i i^{\prime}}^{-1}$.

The following are corollaries of Propositions 5.9 and 5.12.

Theorem 5.13. For $\left[\mathcal{G}_{Y}(B, y)\right]_{\mathbf{T}}$, the following relations hold:

(i) Half periodicity: $\left[y_{\mathbf{i}}\left(u+h^{\vee}+\ell\right)\right]_{\mathbf{T}}=\left[y_{\boldsymbol{\omega}(\mathbf{i})}(u)\right]_{\mathbf{T}}$.

(ii) Full periodicity: $\left[y_{\mathbf{i}}\left(u+2\left(h^{\vee}+\ell\right)\right)\right]_{\mathbf{T}}=\left[y_{\mathbf{i}}(u)\right]_{\mathbf{T}}$.

Theorem 5.14. For $\left[\mathcal{G}_{Y}(B, y)\right]_{\mathbf{T}}$, let $N_{+}$and $N_{-}$denote the total numbers of the positive and negative monomials, respectively, among $\left[y_{\mathbf{i}}(u)\right]_{\mathbf{T}}$ for $(\mathbf{i}, u): \mathbf{p}_{+}$in the region $0 \leq u<2\left(h^{\vee}+\ell\right)$. Then

$$
N_{+}=6 \ell(2 \ell+1), \quad N_{-}=12(3 \ell-2) .
$$

\section{§5.8. Periodicities and dilogarithm identities}

Applying [IIKKN, Theorem 5.1] to Theorem 5.13, we obtain the periodicities:

Theorem 5.15. For $\mathcal{A}(B, x, y)$, the following relations hold:

(i) Half periodicity: $x_{\mathbf{i}}\left(u+h^{\vee}+\ell\right)=x_{\boldsymbol{\omega}(\mathbf{i})}(u)$.

(ii) Full periodicity: $x_{\mathbf{i}}\left(u+2\left(h^{\vee}+\ell\right)\right)=x_{\mathbf{i}}(u)$. 
Theorem 5.16. For $\mathcal{G}(B, y)$, the following relations hold:

(i) Half periodicity: $y_{\mathbf{i}}\left(u+h^{\vee}+\ell\right)=y_{\boldsymbol{\omega}(\mathbf{i})}(u)$.

(ii) Full periodicity: $y_{\mathbf{i}}\left(u+2\left(h^{\vee}+\ell\right)\right)=y_{\mathbf{i}}(u)$.

Theorems 2.5 and 2.6 for $G_{2}$ follow from Theorems 5.5, 5.8, 5.15, and 5.16. Furthermore, Theorem 2.10 for $G_{2}$ is obtained from the above periodicities and Theorem 5.14 as in the $B_{r}$ case [IIKKN, Section 6].

\section{$\S 6$. Mutation equivalence of quivers}

Recall that two quivers $Q$ and $Q^{\prime}$ are said to be mutation equivalent, denoted $Q \sim Q^{\prime}$ here, if there is a quiver isomorphism from $Q$ to some quiver obtained from $Q^{\prime}$ by successive mutations.

Below we present several mutation equivalent pairs of quivers $Q_{\ell}\left(X_{r}\right)$, though the list is not complete at all. For simply laced $X_{r}, Q_{\ell}\left(X_{r}\right)$ is the quiver defined as the square product $\vec{X}_{r} \square \vec{A}_{\ell-1}$ in [Ke, Section 8].

Proposition 6.1. We have the following mutation equivalences of quivers:

$$
\begin{aligned}
Q_{2}\left(B_{r}\right) & \sim Q_{2}\left(D_{2 r+1}\right), \\
Q_{2}\left(C_{3}\right) & \sim Q_{3}\left(D_{4}\right), \\
Q_{2}\left(F_{4}\right) & \sim Q_{3}\left(D_{5}\right), \\
Q_{3}\left(C_{2}\right) & \sim Q_{4}\left(A_{3}\right), \\
Q_{\ell}\left(G_{2}\right) & \sim Q_{\ell}\left(C_{3}\right) .
\end{aligned}
$$

\section{References}

[FZ1] S. Fomin and A. Zelevinsky, Y-systems and generalized associahedra, Ann. of Math. 158 (2003), 977-1018. Zbl 1057.52003 MR 2031858

[FZ2] Cluster algebras IV. Coefficients, Compos. Math. 143 (2007), 112-164. Zbl 1127.16023 MR 2295199

[IIKKN] R. Inoue, O. Iyama, B. Keller, A. Kuniba and T. Nakanishi, Periodicities of T-systems and Y-systems, dilogarithm identities, and cluster algebras I: Type $B_{r}$, Publ. RIMS Kyoto Univ. 49 (2013), 1-42.

[IIKNS] R. Inoue, O. Iyama, A. Kuniba, T. Nakanishi and J. Suzuki, Periodicities of T-systems and Y-systems, Nagoya Math. J. 197 (2010), 59-174. Zbl pre05704436 MR 2649278

[Ke] B. Keller, Cluster algebras, quiver representations and triangulated categories, in Triangulated categories, T. Holm et al. (eds.), London Math. Soc. Lecture Note Ser. 375, Cambridge Univ. Press, 2010, 76-160. Zbl 1215.16012 MR 2681708

[Ki] A. N. Kirillov, Identities for the Rogers dilogarithm function connected with simple Lie algebras, J. Soviet Math. 47 (1989), 2450-2458. Zbl 0685.33014 MR 0947332

[Ku] A. Kuniba, Thermodynamics of the $U_{q}\left(X_{r}^{(1)}\right)$ Bethe ansatz system with $q$ a root of unity, Nucl. Phys. B 389 (1993), 209-244. MR 1202213 
[KN] A. Kuniba and T. Nakanishi, Spectra in conformal field theories from the Rogers dilogarithm, Mod. Phys. Lett. A 7 (1992), 3487-3494. Zbl 1021.81842 MR 1192727

[KNS] A. Kuniba, T. Nakanishi and J. Suzuki, Functional relations in solvable lattice models: I. Functional relations and representation theory, Int. J. Modern Phys. A 9 (1994), 5215-5266. Zbl 0985.82501 MR 1304818

[P1] P.-G. Plamondon, Cluster characters for cluster categories with infinite-dimensional morphism spaces, Adv. Math. 227 (2011), 1-39. Zbl pre05880817 MR 2782186

[P2] Cluster algebras via cluster categories with infinite-dimensional morphism spaces, Compos. Math. 147 (2011), 1921-1954. Zbl 1244.13017 MR 2862067 